\title{
Optimal sensor configuration for ultrasonic guided-wave inspection based on value of information
}

\author{
Sergio Cantero-Chinchilla ${ }^{\mathrm{a}, \mathrm{c}, *}$, Juan Chiachío $^{\mathrm{b}}$, Manuel Chiachío ${ }^{\mathrm{b}}$, Dimitrios Chronopoulos ${ }^{\mathrm{a}}$, Arthur Jones ${ }^{\mathrm{a}}$ \\ a Institute for Aerospace Technology \& The Composites Group, The University of Nottingham, NG7 2RD, United Kingdom \\ $b$ Dept. Structural Mechanics \& Hydraulics Engineering, University of Granada, 18001, Spain \\ c Aernnova Engineering Division S.A., Madrid, 28034, Spain
}

\begin{abstract}
Condition-based maintenance critically relies on efficient and reliable structural health monitoring systems, where the number, position and type of sensors are determined according to rational and principled criteria. This paper proposes the use of the value of information and the relative expected information gain as optimality criteria to determine the best number and positions of sensors, respectively. The proposed methodology is general, but in this paper it is specialized for ultrasonic guided-wave optimal system configuration. Two case studies are used to illustrate the suitability of the proposed methodology in providing the optimal sensor configuration of an ultrasonic guided-wave based structural health monitoring system. The results confirm the value of information as an efficient and rational index to compare among different sensor positioning strategies, while accounting for the underlying modeling and measurement uncertainties. As key contribution, a novel framework that trades-off between amount and cost of information is provided. The results show that geometrically unconstrained sensor configurations are preferred, since they provide a healthier balance between the amount of information and the benefit of such information.
\end{abstract}

Keywords: Optimal sensor configuration, Value of information, Bayesian inverse problem, Ultrasound, Time of flight, SHM

\section{Introduction}

Recent advances in structural health monitoring (SHM) are enabling a progressive transition from scheduled pre3 ventive maintenance to condition-based predictive maintenance in safety-critical industries such as the aerospace 4 industry [1, 2]. These maintenance activities have a profound impact not only on safety but also on cost, since the ser5 vice needs to be suspended during inspection and maintenance. Therefore, higher reliability, availability, and lower 6 operation and maintenance costs are desired outcomes of condition-based maintenance, which highly relies on the 7 amount, coverage, and accuracy of the SHM data. Theoretically, an infinitely reliable system would require an infinite 8 amount of information; however such a theoretical rule finds an exception in practice when features related to the

\footnotetext{
${ }^{*}$ Corresponding Author

Email address: Sergio.CanteroChinchilla1@nottingham.ac.uk (Sergio Cantero-Chinchilla)
} 


\begin{tabular}{|c|c|c|c|}
\hline \multicolumn{4}{|c|}{ Nomenclature } \\
\hline$b(\cdot)$ & benefit function & $n_{s}$ & maximum potential number of sensors \\
\hline$B^{\prime}$ & maximum prior expected benefit & $\mathscr{N}$ & Gaussian distribution \\
\hline$B^{\prime \prime}$ & maximum posterior expected benefit & $P(\cdot)$ & probability \\
\hline BIP & Bayesian inverse problem & $p(\cdot)$ & probability density function \\
\hline BEIG & benefit of the expected information gain & PEB & posterior expected benefit \\
\hline CVI & conditional value of information & $\mathrm{RCI}$ & relative cost of implementation \\
\hline $\mathscr{C}$ & sensor spatial configuration & \multirow{2}{*}{\multicolumn{2}{|c|}{$\begin{array}{l}\mathrm{ToF}_{\mathscr{D}}^{(a-s)} \text { measured ToF between actuator } a \text { and sen- } \\
\text { sor } s\end{array}$}} \\
\hline$D^{(k)}$ & $\begin{array}{l}\text { mean of the hyper-robust model for the } k \text {-th } \\
\text { sensor }\end{array}$ & & \\
\hline D & set of data $D^{(k)}$ for all the sensors & \multirow{2}{*}{\multicolumn{2}{|c|}{$\mathrm{ToF}_{M}^{(a-s)}$ modeled ToF between actuator $a$ and sensor }} \\
\hline $\mathscr{D}^{(k)}$ & signal acquired in the $k$-th sensor & & \\
\hline$e$ & error term in ToF model & & \\
\hline E & Young's modulus & $\gamma$ & threshold parameter for AIMS \\
\hline EVI & Expected value of information & $\gamma_{p}$ & $\begin{array}{l}\text { performance index of the sensor configura- } \\
\text { tion }\end{array}$ \\
\hline$f(n)$ & inverse cost function & $\varepsilon_{\eta}$ & error of damage reconstruction \\
\hline $\mathrm{KL}(\cdot \mid$ & $\begin{array}{l}\text { Kullback-Leibler divergence } \\
\text { optimal configuration under } t\end{array}$ & $v$ & Poisson ratio \\
\hline & pected benefit & $\rho$ & density \\
\hline$n_{o p t}^{\prime \prime}$ & $\begin{array}{l}\text { optimal configuration under the posterior ex- } \\
\text { pected benefit }\end{array}$ & $\Theta$ & $\begin{array}{l}\text { set of possible values of the parameters in the } \\
\text { BIP }\end{array}$ \\
\hline $\mathfrak{N}$ & space of potential configurations & $\boldsymbol{\theta}$ & set of ToF model parameters \\
\hline
\end{tabular}

9 complexity of the monitoring system such as the cost or the weight, are taken into consideration. The latter suggests 10 a trade-off between reliability and complexity for SHM design that needs to be assessed rigorously for optimal SHM

functionality. In the particular case of ultrasonic guided-wave based SHM, the aforementioned trade-off is especially relevant since uncertainty factors such as sensor noise, material property uncertainties, and variations in the acquisition and generation equipment, are well-known sources of complexity which limit the monitoring functionality. For this reason, several authors have recently adopted the concept of value of information [3, 4] to investigate optimal SHM designs based on a healthy and principled balance between the amount and the benefit of the monitoring information. For example, the value of information has been adopted to address the maintenance decision optimization in [5, 6] by using SHM data [7, 8], reliability methods [9], and influence diagrams [10]. Besides, it has also been used to assess the value of SHM systems based on structural risks, integrity management, service life costs, pre-posterior analysis, and Bayesian decision theory [11-14]. In the context of optimal sensor placement, the value of information has been adopted to optimize spatially distributed systems, which allow for information propagation [15], although with known modularity issues [16]. However, none of these contributions to date has focused on ultrasonic guided-wave based 
SHM, precisely where the benefits of this decision-theoretic concept can be fully exploited due to the high reliance of ultrasonic information on both the number and position of the sensors.

In the context of ultrasonic SHM systems design, several approaches have been proposed in the literature to optimize the number of piezoelectric (PZT) sensors and/or their locations in plate-like structures in both active and passive sensing diagnosis using ultrasonic guided-waves. Passive sensing diagnosis techniques consider an arbitrary number of sensors in listening-mode which capture sudden changes in the structure, such as impacts or crack growth. Alternatively, active sensing diagnosis techniques consider the interaction of the sensors with the structure by means of emission and reception of guided waves. In both cases, several attempts have been proposed to optimize the SHM performance (number of sensors and location) through optimization methods such as artificial neural networks combined with (1) genetic algorithms [17-20], whose efficiency has been improved by using the trilateration technique [21], (2) simulated annealing to achieve complete coverage and discrimination [22], and (3) particle swarm optimization [23]. Alternatively, the optimization has been addressed by the use of performance indexes such as the probability of detection [24-27] and the maximization of the area of coverage [28- -31$]$, whereby the ultrasonic guided-wave properties and geometrical complexities are taken into account, as well as features like the influence of faulty sensors [32]. The vast majority of these approaches rely on deterministic assumptions, and hence do not account for the inherent uncertainties associated with ultrasound-based SHM, such as signal noise and uncertain material and ultrasonic parameters. These uncertainties propagate across different models leading to uncertainty in the damage reconstruction, which needs to be quantified for a rigorous optimal sensor location assessment.

Several Bayesian probabilistic approaches for optimal sensor configuration have been proposed in the literature, but for applications other than guided-wave based SHM. For example, the position and/or the number of sensors in structural systems have been optimized by making use of model-based Bayesian inverse problems (BIPs) and probabilistic metrics such as (1) the Shannon-entropy of the posterior distribution [33-37], (2) the Kullback-Leibler (KL) divergence between the prior and posterior distributions [38-42], or (3) the mutual information [43]. In analogous applications, a Bayesian experimental design approach has been proposed, which provides a case-specific utility function, such as the KL divergence [44], whereby the maximization of its probabilistic expectation provides the optimal experimental design [45]. As a general comment, these methods provide rigorous criteria for optimal sensor configuration from an information-theoretic point of view, so that the more sensors the more information gained and the better damage identification. Note that some of these approaches, which focus on the selection of the correlated prediction error models, not only allow the accountability of uncertainties but also provide indications of the optimal number of sensors [36]. However, in practice, decision-theoretic instead of information-theoretic criteria would be preferred, where important variables such as the cost of the monitoring system can be rigorously taken into consideration for optimal SHM system design.

In this paper, a novel and generic methodology for optimal sensor configuration in ultrasonic guided-wave based SHM is provided. To take rigorously into account both information gain and cost-related benefits while accounting for the underlying modeling and measurement uncertainties, the expected value of information is proposed as an op- 
timality criterion. The value of information is defined as the difference between the maximum prior and posterior expected benefits [8], and here it is proposed to be used as an objective function for obtaining the optimal number of sensors. In particular, different sensor configurations with an increasing number of sensors are proposed according to a forward sequential sensor placement [35] algorithm. Then, the optimal sensor position for each of these configurations is determined by maximizing the information gained between the prior and the posterior PDF of the damage identification parameters (e.g., damage position). The computation of the information gain is carried out using a damage localization model based on a robust Bayesian methodology proposed by the authors in [46]. Next, the expected value of information for each of the optimally-located sensor configurations is obtained, so that the optimal configuration is chosen as the one which maximizes the value of information. The methodology is illustrated using two case studies for two different plate-like structures, namely (1) a flat aluminium panel and (2) an aeronautical hat-type stiffened aluminum plate. The optimal sensor configuration is obtained considering the prior information about the possible damage occurrence within a particular bounded area, whereby the data is simulated by using a time of flight model [47]. Then, the effectiveness of the optimal sensor configuration is tested in a particular damage scenario by simulating the structures in Abaqus and reconstructing the damage using the robust Bayesian damage localization methodology provided in [46]. In general, the results show the efficiency of the proposed methodology in obtaining the optimal number and position of sensors, in comparison with pure information based approaches. In particular, the results reveal that non geometrically constrained configurations are preferred from a value of information point of view, since they provide the best trade-off between amount of information and benefit of such information.

The remainder of the paper is organized as follows. Section 2 describes the proposed optimization methodology based on the value of information and the fundamentals of Bayesian damage localization. Section 3 illustrates the methodology through two case studies for two different plate-like structures. A discussion is provided in Section 4 to investigate the influence of the parameters involved in the optimization result and to provide insight on the extensibility of the proposed approach. Finally, Section 5 provides concluding remarks.

\section{Methodology}

\subsection{Optimal sensor configuration based on value of information}

In this section, a methodology based on the concept of value of information [3, 4] is proposed to assess the optimal number of sensors for an ultrasound-based SHM system. In general terms, the value of information quantifies the increment of benefit as a consequence of the information gain about a set of uncertain model parameters $\boldsymbol{\theta}$ (e.g. damage location parameters) when data $\mathbf{D}$ are taken into account.

In mathematical terms, let $\mathfrak{N}=\{1, \ldots, n, \ldots\}$ denote a set of potential sensor configurations and $b(n, \boldsymbol{\theta}): \mathbb{N} \times \mathbb{R}^{n} \boldsymbol{\theta} \rightarrow \mathbb{R}$ a benefit function for the $n$-th sensor configuration, given a set of updatable model parameters $\boldsymbol{\theta} \in \boldsymbol{\Theta} \subset \mathbb{R}^{n} \boldsymbol{\theta}$. Note that the $n$-th sensor configuration implies a layout of $n$ sensors. Let us also denote by $p(\boldsymbol{\theta})$ and $p(\boldsymbol{\theta} \mid \mathbf{D})$ the prior and posterior PDFs of the parameters $\boldsymbol{\theta}$, respectively, with $\mathbf{D}$ being the dataset. In this 
Therefore, by substituting Equations (2), (3) and (1) in Equation (4), the CVI can be expressed as follows:

$$
\begin{aligned}
\operatorname{CVI}(\mathbf{D}) & =\int b\left(n_{o p t}^{\prime \prime}, \boldsymbol{\theta}\right) p(\boldsymbol{\theta} \mid \mathbf{D}) d \boldsymbol{\theta}-\int b\left(n_{\text {opt }}^{\prime}, \boldsymbol{\theta}\right) p(\boldsymbol{\theta}) d \boldsymbol{\theta} \\
& =\int f\left(n_{\text {opt }}^{\prime \prime}\right)\left(\alpha+\log _{2}\left[\frac{p(\boldsymbol{\theta} \mid \mathbf{D})}{p(\boldsymbol{\theta})}\right]\right) p(\boldsymbol{\theta} \mid \mathbf{D}) d \boldsymbol{\theta}-\int f\left(n_{o p t}^{\prime}\right)\left(\alpha+\log _{2}[\underbrace{\left.\left.\frac{p(\boldsymbol{\theta})}{p(\boldsymbol{\theta})}\right]\right) p(\boldsymbol{\theta}) d \boldsymbol{\theta}}_{=1}\right. \\
& =\alpha \int f\left(n_{o p t}^{\prime \prime}\right) p(\boldsymbol{\theta} \mid \mathbf{D}) d \boldsymbol{\theta}+\int f\left(n_{o p t}^{\prime \prime}\right) \log _{2}\left[\frac{p(\boldsymbol{\theta} \mid \mathbf{D})}{p(\boldsymbol{\theta})}\right] p(\boldsymbol{\theta} \mid \mathbf{D}) d \boldsymbol{\theta}-\alpha \int f\left(n_{o p t}^{\prime}\right) p(\boldsymbol{\theta}) d \boldsymbol{\theta}
\end{aligned}
$$

where $p(\boldsymbol{\theta})$ is the prior PDF of model parameters $\boldsymbol{\theta}, \pi(\boldsymbol{\theta}, \mathbf{D})$ is to denote the current PDF of $\boldsymbol{\theta}$, which could be either the prior or the posterior PDFs depending on the availability of the dataset $\mathbf{D}$, and $\log _{2}[\pi(\boldsymbol{\theta} \mid \mathbf{D}) / p(\boldsymbol{\theta})]$ is the information gain between the aforementioned PDFs in terms of bits. In Equation (1), the inverse cost function $f(n)$ can be defined as a generally decreasing and dimensionless function, which gives a measure on how much benefit in terms of cost savings a particular configuration of $n$ sensors provides. The constant $\alpha>0$ introduced in Equation (1) is to represent the basic state of information assumed in the system such that it makes $b(n, \boldsymbol{\theta})=\alpha f(n)$ when $\pi(\boldsymbol{\theta}, \mathbf{D})=$ $p(\boldsymbol{\theta})$, which happens when there is no learning about $\boldsymbol{\theta}$ from data $\mathbf{D}$ and therefore the benefit directly equals to the cost savings.

Next, the concept of maximum prior expected benefit $B^{\prime}$, obtained from the adoption of the optimal configuration $n_{\text {opt }}^{\prime} \in \mathfrak{N}$, is defined as follows [8]:

$$
B^{\prime}=\mathbb{E}_{p(\boldsymbol{\theta})}\left[b\left(n_{o p t}^{\prime}, \boldsymbol{\theta}\right)\right] \quad \leftarrow \quad n_{o p t}^{\prime}=\underset{n \in \mathfrak{N}}{\arg \max } \int b(n, \boldsymbol{\theta}) p(\boldsymbol{\theta}) d \boldsymbol{\theta}
$$

Analogously, the maximum posterior expected benefit $(\mathrm{PEB}) B^{\prime \prime}(\mathbf{D})$ can be obtained as [8]:

$$
B^{\prime \prime}(\mathbf{D})=\mathbb{E}_{p(\boldsymbol{\theta} \mid \mathbf{D})}\left[b\left(n_{\text {opt }}^{\prime \prime}, \boldsymbol{\theta}\right)\right] \quad \leftarrow \quad n_{\text {opt }}^{\prime \prime}=\arg \max _{n \in \mathfrak{N}} \int b(n, \boldsymbol{\theta}) p(\boldsymbol{\theta} \mid \mathbf{D}) d \boldsymbol{\theta}
$$

where the conditioning on $\mathbf{D}$ is to denote that $B^{\prime \prime}$ depends on the data obtained through the PZT sensors. Finally, by subtracting both mathematical expectations, the conditional value of information (CVI) given $\mathbf{D}$ is defined as:

$$
\operatorname{CVI}(\mathbf{D})=B^{\prime \prime}(\mathbf{D})-B^{\prime}
$$

107 Note from the last equation that the inverse cost function is independent of the uncertain parameters $\boldsymbol{\theta}$, therefore 
Equation (5) can be reorganized as follows:

$$
\operatorname{CVI}(\mathbf{D})=\alpha f\left(n_{\text {opt }}^{\prime \prime}\right) \underbrace{\int p(\boldsymbol{\theta} \mid \mathbf{D}) d \boldsymbol{\theta}}_{=1}+f\left(n_{\text {opt }}^{\prime \prime}\right) \underbrace{\int \log _{2}\left[\frac{p(\boldsymbol{\theta} \mid \mathbf{D})}{p(\boldsymbol{\theta})}\right] p(\boldsymbol{\theta} \mid \mathbf{D}) d \boldsymbol{\theta}}_{\mathrm{KL}(p(\boldsymbol{\theta} \mid \mathbf{D}) \| p(\boldsymbol{\theta}))}-\alpha f\left(n_{\text {opt }}^{\prime}\right) \underbrace{\int p(\boldsymbol{\theta}) d \boldsymbol{\theta}}_{=1}
$$

which finally leads to

$$
\operatorname{CVI}(\mathbf{D})=\underbrace{f\left(n_{\text {opt }}^{\prime \prime}\right) \operatorname{KL}(p(\boldsymbol{\theta} \mid \mathbf{D}) \| p(\boldsymbol{\theta}))}_{\text {BEIG }}-\underbrace{\alpha\left[f\left(n_{\text {opt }}^{\prime}\right)-f\left(n_{\text {opt }}^{\prime \prime}\right)\right]}_{\text {RCI }}
$$

where $\operatorname{KL}(p(\boldsymbol{\theta} \mid \mathbf{D}) \| p(\boldsymbol{\theta}))$ denotes the Kullback-Leibler divergence between the posterior and prior PDF of the uncertain parameters $\boldsymbol{\theta}$. In the last equation, the term $f\left(n_{o p t}^{\prime \prime}\right) \operatorname{KL}(p(\boldsymbol{\theta} \mid \mathbf{D}) \| p(\boldsymbol{\theta}))$ can be understood as the benefit of the expected information gain (BEIG), which is always a non-negative value, i.e. BEIG $\in \mathbb{R}_{0}^{+}$. The second part of Equation (7) can be understood as the relative cost of implementation (RCI), since it accounts for the difference between the cost savings of implementing the optimal sensor configuration before and after considering the information from data $\mathbf{D}$. Note that, in practice, not adding any SHM system $(n=0)$ is cheaper than adding it by strictly considering economical factors, due to sensors and hardware costs saving, among others. Therefore, the RCI is positive under the assumption that $f(n)$ is decreasing, i.e. $n_{\text {opt }}^{\prime}<n_{\text {opt }}^{\prime \prime}$ hence $f\left(n_{\text {opt }}^{\prime}\right)>f\left(n_{\text {opt }}^{\prime \prime}\right)$ as exemplified in Figure 1. Observe also that the non-negative constant $\alpha$ defined in Equation (1) avoids the prior expected benefit (Equation (2)) becoming zero, and therefore simpler configurations will be preferred under non-informative (i.e. prior equals posterior) scenarios. In addition, the value of $\alpha$ is defined so that both terms, i.e., BEIG and RCI, are comparable under all possible scenarios, even when the system is not very informative, so that neither one of the RCI nor the BEIG terms individually drives the optimization problem. This interpretation of Equation (7) suggests that the CVI conveys a trade-off between the global benefit of establishing a particular SHM configuration and the cost of actually implementing it. The CVI also provides a decision making index that ranks different strategies considering their suitability in performance and cost. The interpretation of Equation (7) is illustrated with two hypothetical examples below.

Example 1. Let us assume a non-informative SHM system where the posterior PDF is virtually equal to the prior PDF, such that the optimal number of sensors in prior and posterior states are the same, i.e. $n_{o p t}^{\prime}=n_{o p t}^{\prime \prime}$ and thus $f\left(n_{\text {opt }}^{\prime}\right)=f\left(n_{\text {opt }}^{\prime \prime}\right)$, hence RCI $=0$. Moreover, since the SHM system is non-informative, the expected information gain equals zero, and therefore the BEIG equals zero as well. In this extreme example, given that CVI $=0$, no decision about adding or removing sensors could be made based on the current information, hence no change with respect to the prior configuration would be required. Figure $2 \mathrm{a}$ depicts this example.

Example 2. Let us assume a non-informative SHM system such that the optimal number of sensors when data are acquired are $n_{\text {opt }}^{\prime \prime}=n_{\text {opt }}^{\prime} / 2$. In this example, half of the sensor network in the prior or initial state, i.e., $n_{\text {opt }}^{\prime} / 2$, are placed opposite to each other in the plate, as depicted in Figure $2 \mathrm{~b}$. In this situation, let us assume that the posterior information captured by the system is equivalent to the prior information, hence the expected information gain (KL divergence) equals zero, thus BEIG also equals zero. Henceforth, the RCI term turns negative since $f\left(n_{\text {opt }}^{\prime}\right)<f\left(n_{\text {opt }}^{\prime \prime}\right)$, 


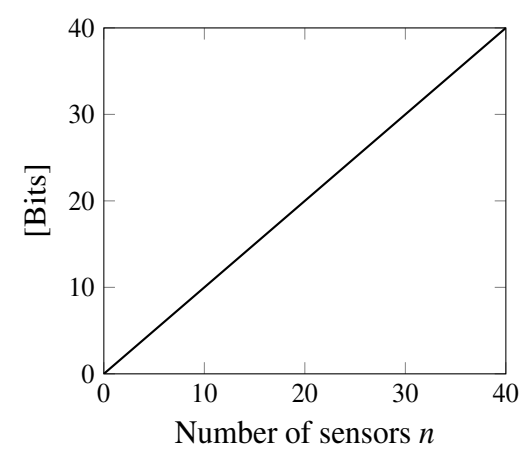

(a) Information gain

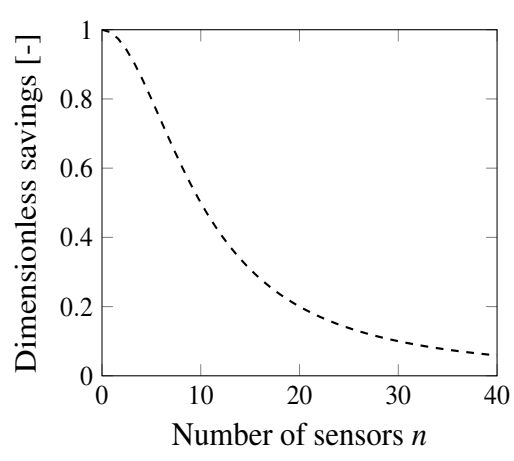

(b) Inverse cost function

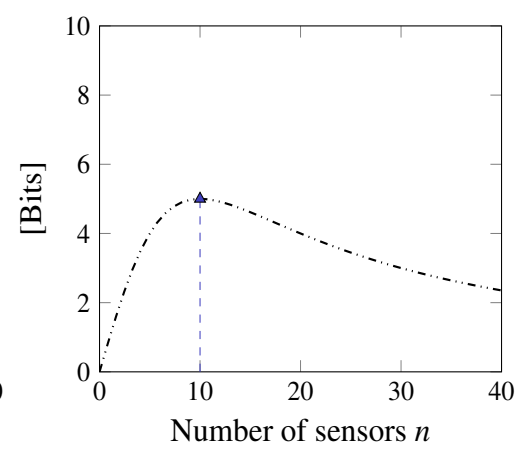

(c) Posterior expected benefit

Figure 1: Example of functions of (a) information gain, (b) dimensionless inverse of cost, and (c) final combination of both functions as an example of Equation (4). It can be observed in (a) that problems that constantly gain information when the number of sensors increases, can be converted into a normal optimization problem (like in (c)) by the introduction of a dimensionless cost function (b).

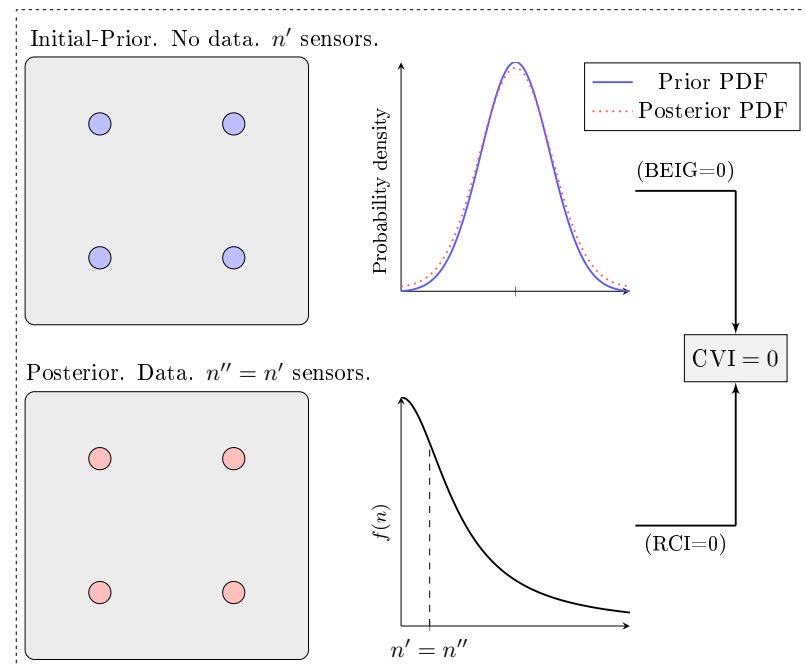

(a) Example 1

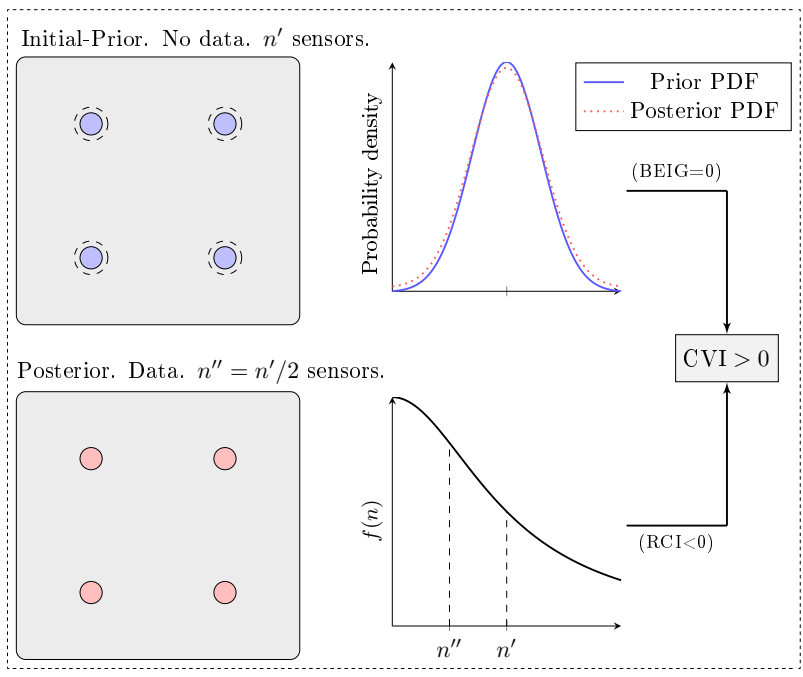

(b) Example 2

Figure 2: Graphical representation of Examples 1 and 2 about the influence of using the proposed CVI-based formulation in the decision of number of sensors. For Example 1, no action would be taken, thus the prior distribution of sensors would be maintained. For Example 2, even when the $\mathrm{BEIG}=0$, the proposed $\mathrm{CVI}>0$ formulation supports the option with a smaller number of sensors. Dashed circles represent the sensors in the opposite side of the plate.

which leads to CVI $>0$. This hypothetical example shows that the proposed formulation propitiates simpler and cheaper SHM systems under non-informative scenarios.

\subsubsection{Expected value of information}

The CVI criterion presented above formulates the optimization problem for a particular set of data $\mathbf{D}$, i.e., it implicitly implies that damage is located at a particular position. However, in practice, the optimal sensor configuration problem requires addressing a large enough set of damage scenarios, hence a space of datasets $\mathfrak{D} \ni \mathbf{D}$ instead of a particular $\mathbf{D}$ needs to be considered. Note that at the design stage, experimental data are not typically available and 
therefore the data are simulated using a model and prior knowledge of the model parameters. Thus, a mathematical expectation is applied to Equation (7) to obtain the expected value of information (EVI) over the space of datasets $\mathfrak{D}$, as follows:

$$
\mathrm{EVI}=\int_{\mathfrak{D}}\left[f\left(n_{\text {opt }}^{\prime \prime}\right) \operatorname{KL}(p(\boldsymbol{\theta} \mid \mathbf{D}) \| p(\boldsymbol{\theta}))\right] p(\mathbf{D}) d \mathbf{D}-\int_{\mathfrak{D}} \alpha\left[f\left(n_{o p t}^{\prime}\right)-f\left(n_{o p t}^{\prime \prime}\right)\right] p(\mathbf{D}) d \mathbf{D}
$$

Observe that the first term of Equation 8 involves a double multidimensional integral that cannot be solved analytically in most of the cases. Therefore, it is numerically approximated using the Monte Carlo (MC) method as follows [38, 41, 48]:

$$
\mathrm{EVI} \approx f\left(n_{\text {opt }}^{\prime \prime}\right) \frac{1}{N_{\text {out }}} \sum_{m=1}^{N_{\text {out }}}\left[\log _{2} p\left(\mathbf{D}_{(m)} \mid \boldsymbol{\theta}_{(m)}\right)-\log _{2}\left(\frac{1}{N_{\text {in }}} \sum_{k=1}^{N_{\text {in }}} p\left(\mathbf{D}_{(m)} \mid \boldsymbol{\theta}_{(k)}\right)\right)\right]-\alpha\left[f\left(n_{\text {opt }}^{\prime}\right)-f\left(n_{\text {opt }}^{\prime \prime}\right)\right]
$$

where $\boldsymbol{\theta}_{(m)}$ is a sample drawn from the prior distribution $p(\boldsymbol{\theta})$ and $\mathbf{D}_{(m)}$ is a sample dataset drawn from the likelihood distribution $p\left(\mathbf{D} \mid \boldsymbol{\theta}=\boldsymbol{\theta}_{(m)}\right)$ (refer to Equation (12) below). Thus, Equation (9) is adopted here as optimality criterion to obtain the optimal sensor configuration considering an area of possible damage locations. Further implementation details of the EVI criterion and the adopted search algorithm are provided hereinafter in Section 2.3 .

\subsection{Optimal sensor placement: Bayesian damage localization}

The previous section presented a rational fitness function to obtain the optimal number of sensors using the EVI, which requires updated information about the model parameters (e.g. damage coordinates) given a particular dataset $\mathbf{D}_{(m)}=\mathbf{D}$. This section describes the fundamentals of the Bayesian inverse problem whereby model parameters are estimated.

The problem of damage localization is addressed by a model-based BIP using an ellipse-based time-of-flight (ToF) model [47], which was previously published by the authors in [46]. The ToF is an ultrasonic signal feature widely adopted by both practitioners and researchers due to its efficiency in providing information about material properties and damage localization by post-processing scattered signals. To estimate the ToF from scattered signals, several timefrequency (TF) representation techniques are available in the literature [49-52]. In this work, a recently published hyper-robust approach is adopted to obtain a robust ToF estimate resulting from using not just a particular TF model class based on modeler's choice, but an overall set of candidate models according to their relative plausibility [46].

In particular, given a TF model, the ToF can be obtained as the difference between the time to receive the first energy peak of the excitation signal and the one from the scattered signal. From a theoretical point of view, the ToF information of the scattered signals can be obtained as follows [53]:

$$
\operatorname{ToF}^{(a-s)}=\frac{\sqrt{\left(X_{d}-X_{a}\right)^{2}+\left(Y_{d}-Y_{a}\right)^{2}}}{V_{a-d}}+\frac{\sqrt{\left(X_{d}-X_{s}\right)^{2}+\left(Y_{d}-Y_{s}\right)^{2}}}{V_{d-s}}
$$

where $\left(X_{d}, Y_{d}\right) \in \mathbb{R}^{2}$ are the coordinates of the damage position, $\left(X_{a}, Y_{a}\right) \in \mathbb{R}^{2}$ are the actuator transducer coordi- 
nates, $\left(X_{s}, Y_{s}\right) \in \mathbb{R}^{2}$ are the coordinates of one particular sensor transducer, and $V_{a-d}$ and $V_{d-s}$ are the wave propagation velocities of the actuator-damage and damage-sensor paths, respectively ${ }_{1}^{1}$ From this standpoint, the unknown model parameters of interest are $\boldsymbol{\theta}=\left\{X_{d}, Y_{d}, V\right\}$ so, under the assumption of perfect sensor, if an exact value of $\boldsymbol{\theta}^{*}$ is known, then the measured ToF and the modeled one using Equation 10 would be identical; mathematically: $\operatorname{ToF}_{\mathscr{D}}^{(a-s)} \equiv \operatorname{ToF}_{M}^{(a-s)}\left(\boldsymbol{\theta}^{*}\right)$. However, in practice there are uncertainties due to signal measurement errors, partially unknown material properties, and the uncertainty about the validity of the ToF model itself, which make the last identity seldom observed in real-world scenarios. Thus, a more appropriate and rigorous approach involves assuming the existence of these modeling uncertainties and quantifying them, as follows:

$$
\operatorname{ToF}_{\mathscr{D}}^{(a-s)}=\operatorname{ToF}_{M}^{(a-s)}(\boldsymbol{\theta})+e
$$

where $e \in \mathbb{R}$ is an error term enclosing the uncertainty which accounts for the discrepancy between $\operatorname{ToF}_{M}^{(a-s)}$ and $\mathrm{ToF}_{\mathscr{D}}^{(a-s)}$. By the Principle of Maximum Information Entropy [54, 55], this error term can be conservatively assumed to be modeled as a zero-mean Gaussian distribution with standard deviation $\sigma_{e} \in \mathbb{R}$. Thus, Equation (11) can be rewritten probabilistically as:

$$
p\left(\operatorname{ToF}_{\mathscr{D}}^{(a-s)} \mid \boldsymbol{\theta}\right)=\left(2 \pi \sigma_{e}^{2}\right)^{-\frac{1}{2}} \exp \left(-\frac{1}{2}\left(\frac{\operatorname{ToF}_{\mathscr{D}}^{(a-s)}-\mathrm{ToF}_{M}^{(a-s)}(\boldsymbol{\theta})}{\sigma_{e}}\right)^{2}\right)
$$

which provides a probabilistic measure about the similarity between $\operatorname{ToF}_{\mathscr{D}}^{(a-s)}$ and $\operatorname{ToF}_{M}^{(a-s)}(\boldsymbol{\theta})$ for a particular value of $\boldsymbol{\theta}$. The last equation is commonly known as the likelihood function for model parameter $\boldsymbol{\theta}$.

Nevertheless, our interest precisely lies in the reciprocal information, i.e., to determine the values of $\boldsymbol{\theta}$ among the set of values $\Theta \subset \mathbb{R}^{n_{\boldsymbol{\theta}}}$ which lead to models that more likely satisfy the ideal identity $\mathrm{ToF}_{\mathscr{D}}^{(a-s)} \equiv \mathrm{ToF}_{M}^{(a-s)}(\boldsymbol{\theta})$. This inverse problem can be formulated by Bayes' Theorem [56, 57], as:

$$
p(\boldsymbol{\theta} \mid \mathbf{D})=\frac{p(\mathbf{D} \mid \boldsymbol{\theta}) p(\boldsymbol{\theta})}{p(\mathbf{D})}
$$

where $\mathbf{D}=\left\{D^{(1)}, \ldots, D^{(k)}, \ldots, D^{(n)}\right\}$ is the set of ToF data corresponding to the set of $n$ sensors and a spatial configuration ${ }^{2} \mathscr{C}^{n}, p(\boldsymbol{\theta})$ is the prior PDF of the model parameters, and $p(\mathbf{D} \mid \boldsymbol{\theta})$ is the likelihood function for the set of data D. Assuming stochastic independence of the measurements, the likelihood $p(\mathbf{D} \mid \boldsymbol{\theta})$ can be expressed probabilistically as $p(\mathbf{D} \mid \boldsymbol{\theta})=\prod_{k=1}^{n} p\left(D^{(k)} \mid \boldsymbol{\theta}\right)$, where each factor $p\left(D^{(k)} \mid \boldsymbol{\theta}\right)$ is given by Equation (12). Finally, the term $p(\mathbf{D})$ refers to the evidence [54] of the data under the model specified by $\boldsymbol{\theta}$. This term, which acts as a normalizing constant within the Bayes' theorem, can be bypassed through sampling using Markov Chain Monte Carlo (MCMC) methods [58].

\footnotetext{
${ }^{1}$ Note that these velocities are the same under the assumption of isotropic materials and damage concentrated within a bounded region, where $V=V_{a-d}=V_{d-s}$.

${ }^{2}$ The conditioning on $\mathscr{C}^{n}$ has been omitted from $p(\boldsymbol{\theta} \mid \mathbf{D})$ given that it is intrinsically contained in $\mathbf{D}$, since a particular configuration univocally implies a dataset, i.e., $\mathscr{C}^{n} \rightarrow \mathbf{D}$.
} 


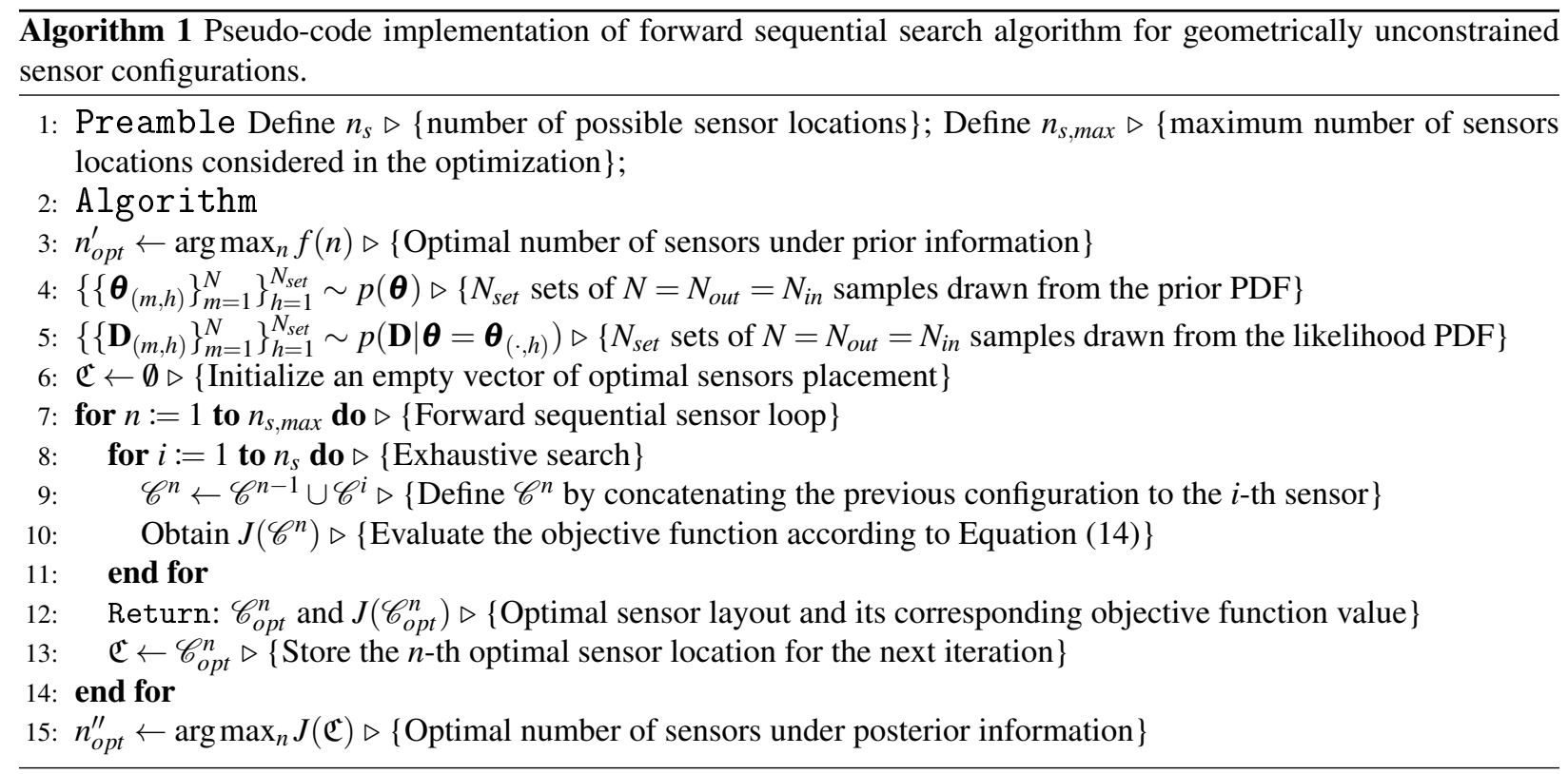

\subsection{Algorithmic implementation}

In this section, a forward sequential algorithm is used to solve the optimal sensor configuration problem over two main variables: (1) number and (2) position of the sensors, as proposed by [35, 36]. Therefore, the algorithm provides the optimal number of sensors $n_{o p t}^{\prime \prime}$ and their optimal spatial configuration $\mathscr{C}_{o p t}^{n}$, whereby the EVI is finally obtained. In this algorithm, the optimal sensor placement problem is proposed to be solved through an exhaustive search methodology, which is performed by exploring all the possible sensor locations within a discrete grid, given that a relatively small search space is considered. The objective function $J\left(\mathscr{C}^{n}\right): \mathbb{N} \rightarrow \mathbb{R}$ for the optimization problem is given by the average of the EVI (Equation (9) for each spatial configuration $\mathscr{C}^{n}$, as follows:

$$
\begin{aligned}
J\left(\mathscr{C}^{n}\right) & =f(n) \frac{1}{N_{\text {set }}} \sum_{h=1}^{N_{\text {set }}}\left\{\frac{1}{N_{\text {out }}} \sum_{m=1}^{N_{\text {out }}}\left[\log _{2} p\left(\mathbf{D}_{(m, h)} \mid \boldsymbol{\theta}_{(m, h)}, \mathscr{C}^{n}\right)-\log _{2}\left(\frac{1}{N_{\text {in }}} \sum_{k=1}^{N_{\text {in }}} p\left(\mathbf{D}_{(m, h)} \mid \boldsymbol{\theta}_{(k, h)}, \mathscr{C}^{n}\right)\right)\right]\right\}- \\
& -\alpha\left[f\left(n_{\text {opt }}^{\prime}\right)-f(n)\right]
\end{aligned}
$$

where $\mathscr{C}^{n}$ is the sensor configuration variable that controls which sensors are active, their location, and therefore what ToF data $\mathbf{D}$ are used to calculate $J\left(\mathscr{C}^{n}\right)$. To efficiently address the expectation of the KL divergence (recall Eq. (9)), we reuse the $N_{\text {out }}=N_{\text {in }}$ prior samples in both the inner and outer sums of Equation (14) at the cost of a small increment in the bias of the estimator [38, 41]. Nevertheless, the computation of the KL divergence implies a numerical error inversely proportional to the number of samples, leading to stochastic uncertainty in the value of the KL divergence. To reduce such stochasticity, the objective function is averaged over $N_{\text {set }}$ sets of samples for each configuration $\mathscr{C}^{n}$, as shown in Equation (14).

Furthermore, note that $n_{\text {opt }}^{\prime}$ in Equation (14) is obtained using Equation (2) and that the independence of the prior 
expected benefit on the sensor configuration makes it only dependent on the inverse cost function. Thus, $n_{o p t}^{\prime}$ implies the most economically beneficial amount of sensors given that $f(n)$ is decreasing, regardless of their position.

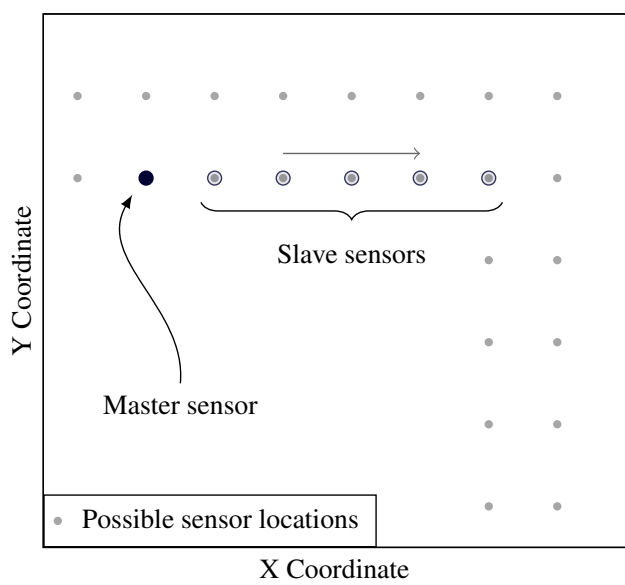

(a) Sensor distribution using 1 array and 6 sensors

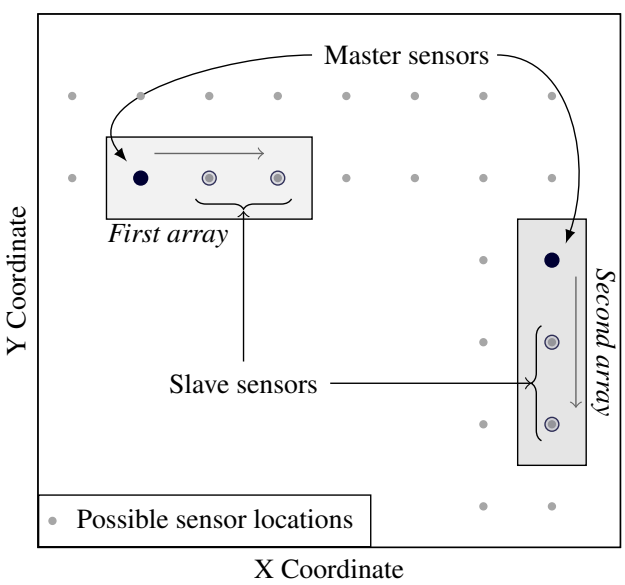

(b) Sensor distribution using 2 arrays and 6 sensors

Figure 3: Illustration of sensor positioning strategies for 1 and 2 arrays. In panel (a), 1 array configurations where one master sensor is optimally placed, while the slave sensors are automatically placed next the master. Panel (b) depicts the case of 2 arrays of sensors, where the first array is optimally placed and its position stored to search then the optimal position of the second one.

Furthermore, observe that the search of the optimal number of sensors implies a heavy computational problem involving $n_{s} ! / n !\left(n_{s}-n\right)$ ! potential configurations to be explored. For example, for $n_{s}=40$ possible sensor locations and $n=5$ sensors, the number of possible configurations would be $40 ! / 5 ! 35 !=658,008$. To avoid such computational complexity, several strategies can be applied to reduce the search space. In this work, the forward sequential search approach [35] is adopted, as shown in Algorithm 11. consisting of running an exhaustive search for one individual sensor (e.g., $n$ ) having stored the optimal locations of the previous $n-1$ sensors, thus limiting the search space. Following this methodology, the total number of configurations reduces to $n\left(2 n_{s}-n+1\right) / 2$. Taking the same numerical example with $n_{s}=40$ and $n=5$, the number of possible configurations would be $5(80-5+1) / 2=190$ instead of 658,008 using the former method. Note that a suboptimal configuration is expected to be obtained, since the sequential search method does not necessarily provide the global optimal solution. However, the suboptimal configuration can be assumed to be effective and robust [59, 60].

From this standpoint, it is important to remark that Algorithm 1 provides a methodology to obtain geometrically unconstrained ("open”) optimal sensor configurations. However, these may find a limitation in practice when installation issues such as the routing of the wires are taken into account. Therefore, more practical sensor configurations constrained to linear arrays of sensors [61, 62] are typically preferred for their ease of installation and maintenance. These sensor configurations using one or two arrays of sensors can be assessed by slightly modifying Algorithm 1 as follows: For one array of $n$ sensors, only the $n$-th sensor (master) position is optimized, considering that the remaining $n-1$ sensors (slave) are placed either on the left or right side of the master one, as depicted in Figure 3a Note for this configuration the search space reduces as long as $n$ increases. In case of two arrays of $n$ sensors, the optimization 
is carried out in two steps, (1) the first array of $n / 2$ sensors is optimally placed, as explained before, and its position is stored, and (2) the optimal position of the second array of $n / 2$ sensors is addressed considering the first one fixed, as shown in Figure $3 \mathrm{~b}$ The search space reduces even faster for two array configurations since only even numbers of sensors $n$ are considered to form the two arrays.

\section{Case Studies}

The proposed methodology to obtain the optimal sensor configuration based on EVI is exemplified herein using two case studies. Section 3.1 illustrates the methodology for a square aluminum plate. Finally, Section 3.2 provides a comparison between open and array configurations for an aeronautical hat-type stiffened plate.

\subsection{Sensor optimization in a square metallic plate}

This case study deals with the problem of optimal sensor configuration for a $30 \mathrm{~cm} \times 30 \mathrm{~cm} \times 0.2 \mathrm{~cm}$ aluminum 2024-T351 plate. The prior information of the model parameters $\boldsymbol{\theta}$ is represented as uniform distributions for the damage position parameters $\left(X_{d}, Y_{d}\right)$ and as a Gaussian distribution for the velocity $V$, as follows: $p\left(X_{d}\right)=\mathscr{U}(-6,6)$, $p\left(Y_{d}\right)=\mathscr{U}(-6,6)$, and $p(V)=\mathscr{N}(5400,60)$, with position and wave velocity units expressed in $[\mathrm{cm}] \mathrm{and}[\mathrm{m} / \mathrm{s}]$ respectively. Note that the origin of coordinates is located at the center of the plate and the wave propagation velocity refers to the symmetric-0 guided-wave mode at a frequency $300 \mathrm{kHz}$. To address the optimal sensor configuration, a grid of possible sensor locations is defined as two concentric squares of $12 \mathrm{~cm}$ and $18 \mathrm{~cm}$ of side respectively, resulting in a total of 40 possible locations. The area of possible damage occurrence is represented in blue color in Figure $4 \mathrm{~b}$. The actuator is considered to be fixed at the origin of coordinates, i.e., the center of the plate. The optimization is addressed by using Algorithm 1 with $N_{\text {set }}=1000$ and $N_{\text {out }}=N_{\text {in }}=1000$ (recall Eq. (14p).

The results for this case study are shown in Figure 4 As evident from Figure $4 \mathrm{a}, J\left(\mathscr{C}_{\text {opt }}^{n}\right)$ reaches a global maximum using a configuration of 3 sensors, provided an inverse cost function $f_{0}(n)$ given by:

$$
f_{0}(n)=100 /\left(n^{2}+100\right)
$$

The corresponding EVI value is 5.4875 [bits], adopting $\alpha=1$ in Equation (9), i.e., assuming that both the RCI and the information members in Equation (14) are equally important. The optimal sensor distribution for this configuration is shown in Figure $4 \mathrm{~b}$ Note that the optimal sensor positions (in red) result to be symmetric with respect to the center of the plate (where the actuator is located), and they are wholly contained within the inner square, i.e., closer to the area of possible damage occurrence.

\subsubsection{Verification of the optimal sensor configuration}

The performance of the optimal sensor configuration, as depicted in Figure 4b, is tested against simulated data for a specific damage within the area of possible damage occurrence. To this end, the plate is simulated in Abaqus 


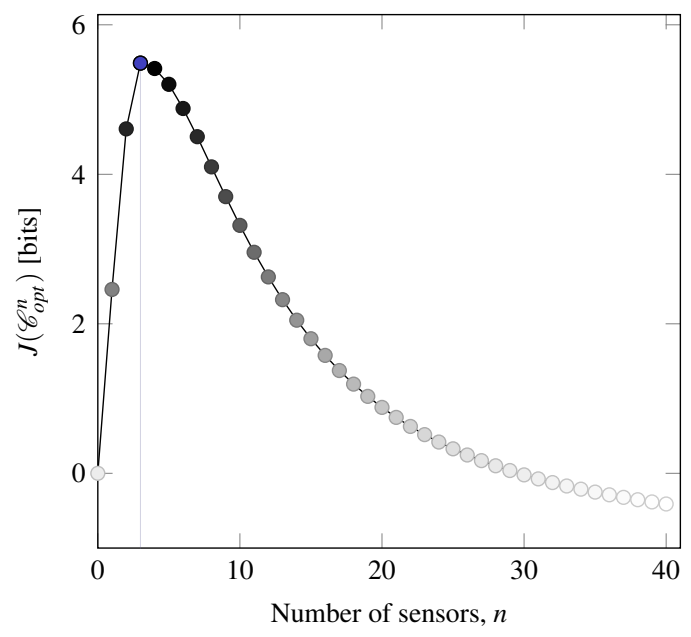

(a) Value of information

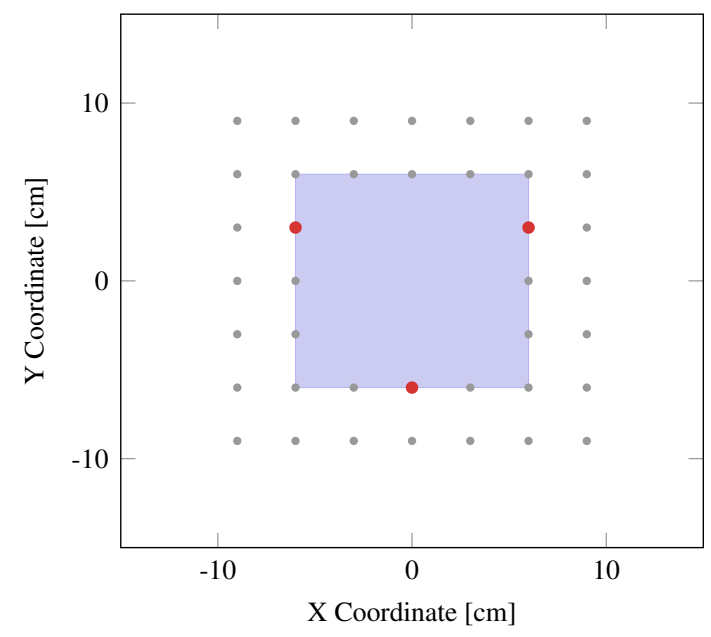

(b) Optimal sensor distribution

Figure 4: Estimator of the EVI (Eq. 14) in panel (a) and the corresponding optimal sensor configuration with $n_{\text {opt }}^{\prime \prime}=3$ sensors in (b), where the possible sensor locations (gray dots) and the optimal locations (red dots) are shown. The blue area denotes the area of possible damage occurrence.

(Figure 5a) using C3D8R (8-node linear brick, reduced integration, hourglass control) solid elements [63] with mesh size $0.05 \mathrm{~cm}$ and mechanical properties $\rho=2780 \mathrm{~kg} / \mathrm{m}^{3}, E=73.1 \mathrm{GPa}$, and $v=0.33$ (refer to the Nomenclature). The mesh size is determined so that at least 10 nodes are contained per wavelength, and the chosen time step is obtained so that a disturbance cannot propagate through a grid spacing during one time step [64, 65]. The simulated damage is represented as a square $0.2 \mathrm{~cm} \times 0.2 \mathrm{~cm}$ hole, centered at $(3.5,2.5) \mathrm{cm}$, considering the center of the plate as the origin of coordinates, as shown in Figure $5 \mathrm{~b}$. To simulate the wave propagation, an exciting force is applied at the center of the plate in the perpendicular direction, consisting of a 5 cycle sine tone burst centered at a frequency $300 \mathrm{kHz}$. The guided-waves are acquired at the three optimal sensor positions highlighted in red in Figure $4 \mathrm{~b}$ and subtracted before and after damage to obtain the scattered signals. Then, the robust ToF estimation methodology previously developed in [46] is applied. The robust ToF data $\mathbf{D}=\left\{D^{(1)}, \cdots, D^{(n)}\right\}$ obtained for each of the optimal sensors are then used as input data for the Bayesian damage localization problem described in Section 2.2, whereby the posterior PDFs of the model parameters $\boldsymbol{\theta}=\left\{X_{d}, Y_{d}, V\right\}$ are obtained. The prior knowledge of the model parameters is assumed to be equal to the one used for the optimization process, described in the Section 3.1. To numerically solve the Bayesian inverse problem of damage localization, the asymptotic independence Markov sampling (AIMS) algorithm [66, 67] is applied with a threshold value $\gamma=1 / 2$, using 50,000 samples per annealing level, using a Gaussian PDF as proposal distribution, i.e. $q\left(\theta^{\prime} \mid \theta\right)=\mathscr{N}(\theta, \sigma)$, where $\sigma$ is the standard deviation of the Metropolis-Hastings random walk. In this problem, $\sigma$ is selected such that the acceptance rate lies within the suggested interval $[0.2,0.4][68,-70]$.

The posterior information of the damage parameters $\boldsymbol{\theta}=\left\{X_{d}, Y_{d}, V\right\}$ is illustrated in Figures $5 \mathrm{c}$ and $5 \mathrm{~d}$. It can be observed that the damage position is remarkably well reconstructed using the optimal sensor configuration. In contrast, the uncertainty of the reconstructed wave propagation velocity is higher as shown in Figure 5d. A larger variability of 


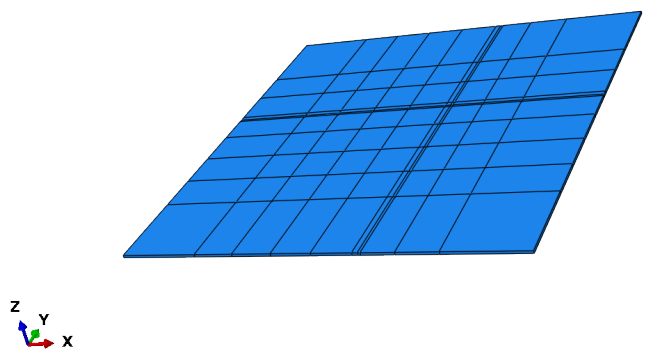

(a) Abaqus model

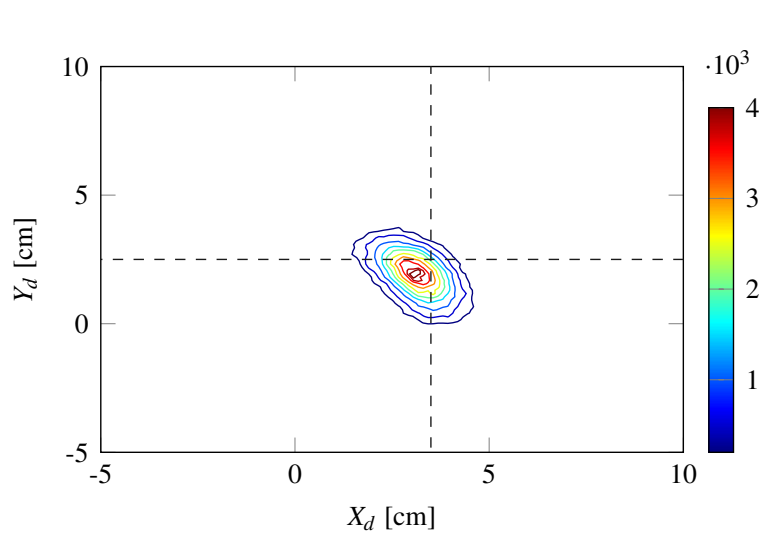

(c) Damage position reconstruction

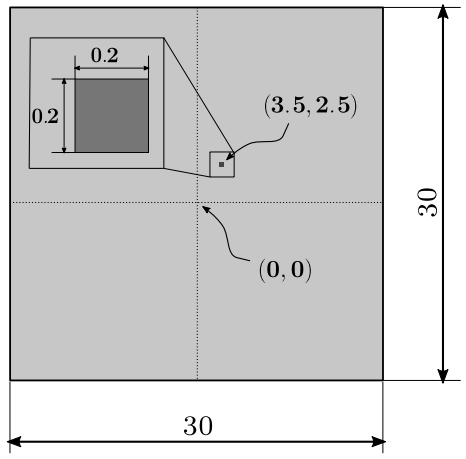

(b) Origin and damage coordinates in $\mathrm{cm}$

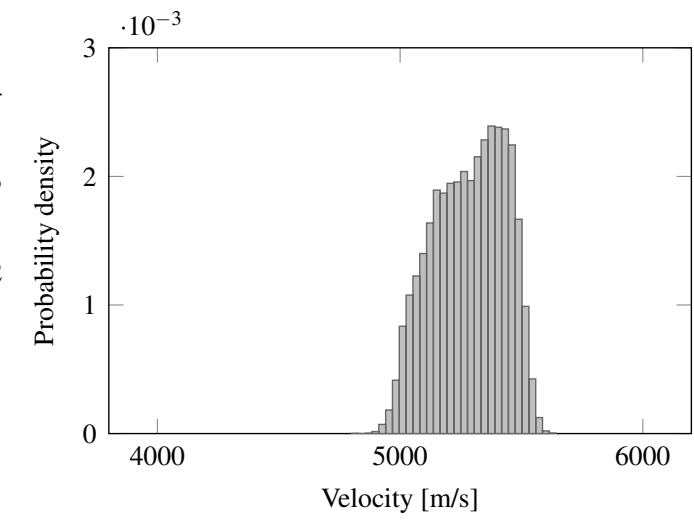

(d) Posterior velocity distribution

Figure 5: Abaqus model in panel (a), dimensions of the plate and location of the simulated damage in panel (b), joint posterior distribution of the damage coordinates with their associated probability densities in the color bar along with the center of the real damage represented by the intersection of both dashed lines in panel (c), and the velocity reconstruction in panel (d).

the ToF data at the optimal sensors is identified as the reason for such relatively high uncertainty in the velocity, which may come from potential mode mixture issues between both anti-symmetric and symmetric 0 guided waves modes, namely A0 and S0 modes, respectively.

\subsection{Sensor optimization in aeronautical panel}

The aim of this case study is to investigate the applicability of the proposed methodology using a more complex and realistic structure. In particular, a $50 \mathrm{~cm} \times 50 \mathrm{~cm} \times 0.2 \mathrm{~cm}$ plate with top-hat section stiffeners, commonly used in aeronautical structures, is adopted for this case study. Here, the origin of coordinates is located at the center of the left plate's edge and the actuator is placed at the center of the plate, i.e., $(25,0)[\mathrm{cm}]$. The prior information of the model parameters is given by $p\left(X_{d}\right)=\mathscr{U}(5,45), p\left(Y_{d}\right)=\mathscr{U}(-10,10)$, and $p(V)=\mathscr{N}(2800,60)$, with position and wave velocity units expressed in $[\mathrm{cm}]$ and $[\mathrm{m} / \mathrm{s}]$ respectively. Note that the velocity prior PDF is centered at $2800 \mathrm{~m} / \mathrm{s}$, which corresponds to the group velocity of the ultrasonic guided-wave mode A0 at a frequency $300 \mathrm{kHz}$. The search space is represented by eight rows of 39 possible sensor locations at 


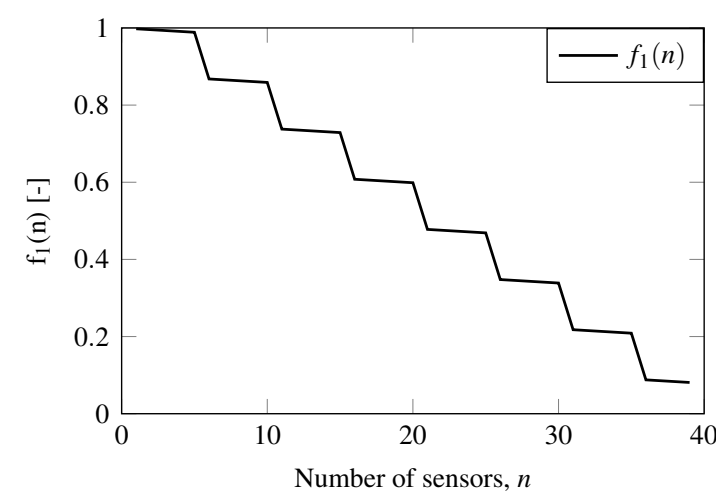

(a) Inverse cost function

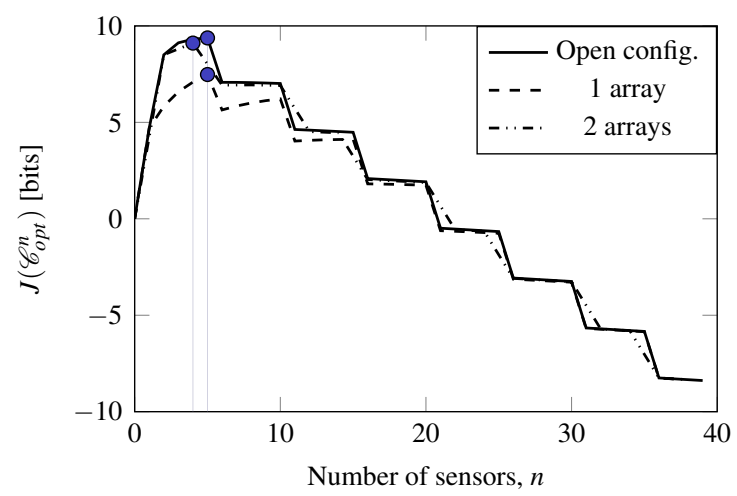

(b) Value of information

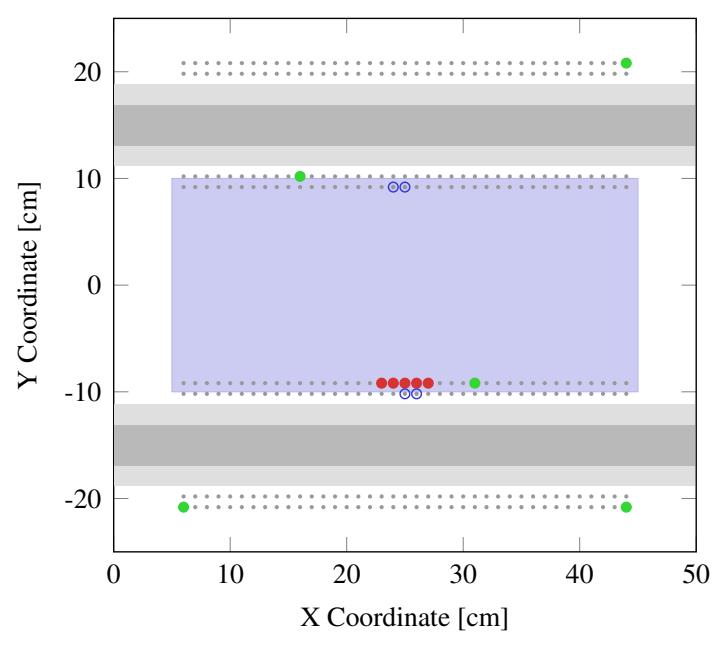

(c) Optimal sensor distributions

Figure 6: Inverse cost function $f_{1}(n)$ in panel (a). In (b), the estimator of the EVI (Eq. (14) for the different positioning configurations is depicted. Panel (c) shows their corresponding optimal sensor layouts in green, red, blue, and gray dots for open configuration, one-array configuration, twoarray configuration, and search space, respectively. The blue rectangle represents the area of possible damage occurrence with uniform probability.

For this case study, a comparison between different sensor placement strategies, i.e., (1) non geometrically constrained "open" configuration, (2) sensor positioning in 1 array, and (3) sensor positioning over 2 separated arrays, is carried out. Figure 6a depicts the adopted inverse cost function, whilst Figure 6b depicts the objective function output $J\left(\mathscr{C}_{o p t}^{n}\right)$ (recall Eq. (14)) as a function of the number of sensors and their respective optimal number of sensors for each type of configuration. The optimal number of sensors results to be $n_{o p t}^{\prime \prime}=5$ for both the open and the one-array configurations $(\mathrm{EVI}=9.2874$ [bits] and EVI = 7.3896 [bits], respectively), whereas the two-array configuration leads to 4 sensors $(\mathrm{EVI}=9.0416$ [bits]), assuming $\alpha=10$ to emphasize the cost over the relative gain of information. In view of Figure $6 \mathrm{~b}$, both the open configuration and the one using 2 arrays result to be the most valuable, and hence 
the preferred ones under the EVI criterion. This ranking is also supported by the robust BEIG and RCI terms (Eq. (7) and (9) in Table 1. In particular, observe that even when the RCI for the open configuration is higher than the RCI of the two-array configuration, the first one is preferred since it provides a relatively higher and robust BEIG.

Table 1: Decomposition of EVI into the robust BEIG and RCI terms (Eq. 97) for the three positioning strategies.

\begin{tabular}{lcccc}
\hline Configurations & BEIG [bits] & RCI [bits] & EVI [bits] & No sensors \\
\hline Open config. & 9.3774 & 0.0900 & 9.2874 & 5 \\
\cline { 2 - 5 } $\begin{array}{l}\text { One array } \\
\text { Two arrays }\end{array}$ & 7.4796 & 0.0900 & 7.3896 & 5 \\
\hline
\end{tabular}

As a general comment, the open configuration is able to reconstruct the damage more accurately due to its flexibility. However, in real world engineering applications, factors such as the routing options of the cables introduce limitations and complexity that may prevent us from the use of sensor open configurations. Accordingly, the two-array configuration would be the preferred one based on the value of information criterion, as shown in Table 1 . Finally, Figure 6c shows the optimal sensor layouts for each of the three positioning strategies.

\subsubsection{Verification of the optimal sensor configuration}

The resulting optimal sensor configurations in Section 3.2 are tested using a simulated damage scenario considering a hole in the metallic plate. To this end, an Abaqus model, shown in Figure $7 \mathrm{a}$ is developed to obtain the simulated guided waves whereby the robust ToF is estimated and the Bayesian damage localization is addressed. Here, the stiffeners are assumed to be perfectly bonded to the base plate whereas the damage is simulated as a $0.2 \mathrm{~cm} \times 0.4 \mathrm{~cm}$ hole located at $(35.1,3) \mathrm{cm}$, considering the origin of coordinates at the center of one plate's edges, as shown in Figure 7b. S4R (4-node doubly curved thin or thick shell, reduced integration, hourglass control, finite membrane strains) shell elements [63] adopted with a mesh size of $0.05 \mathrm{~cm}$; therefore, only anti-symmetric modes are captured, further simplifying the robust ToF identification since no mode mixture is expected to arise at a frequency $300 \mathrm{kHz}$. The guided waves are generated with a 5 cycle sine tone burst centered at the aforementioned frequency applied in the perpendicular direction at the center of the plate. The plate and stiffeners dimensions are depicted in Figure $7 \mathrm{~b}$. The material is aluminum 2024-T351, thus the mechanical properties (Young's modulus $E$ and Poisson ratio $v$ ) are the specified for the previous case study in Section 3.1.1. The prior knowledge of the model parameters remains the same than the specified in Section 3.2

The reconstruction of the damage position corresponding to the optimal configurations is depicted in Figures $7 \mathrm{c}$ to $7 \mathrm{e}$ As evident from the damage localization results, both the open configuration as well as the configuration with two arrays reconstruct the damage position remarkably well, while the configuration using one array provides higher uncertainty about the damage position. Note that the single array configuration would need comparatively more sensors than either the open or double array configurations to achieve the same level of accuracy. Such behavior is also 


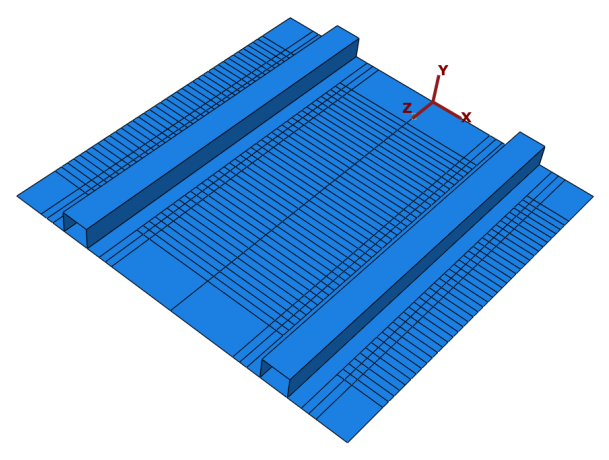

(a) Abaqus model

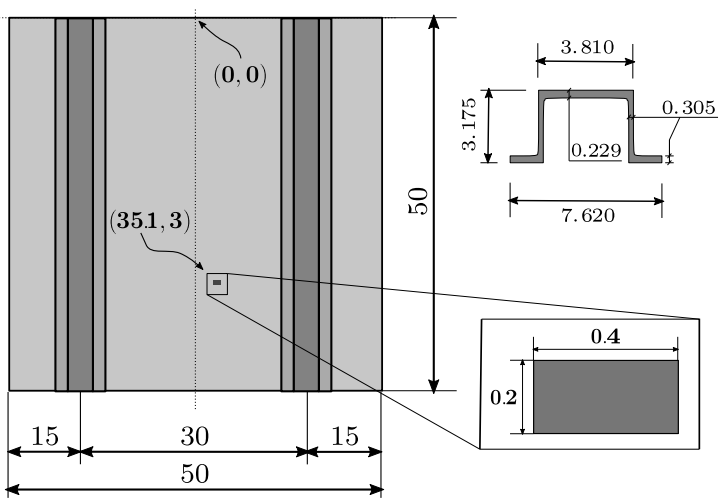

(b) Basic dimensions in $\mathrm{cm}$
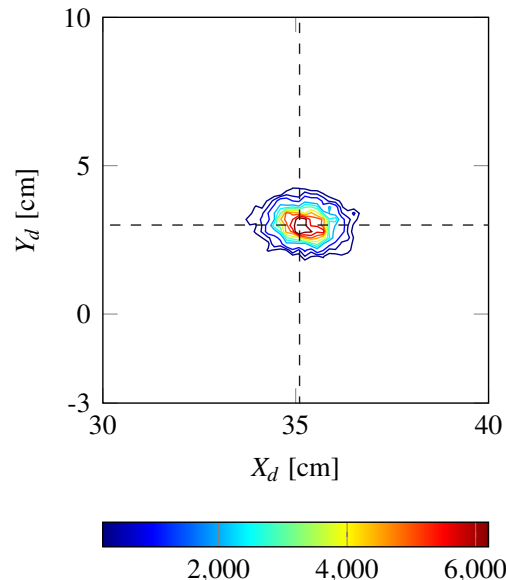

(c) Open configuration

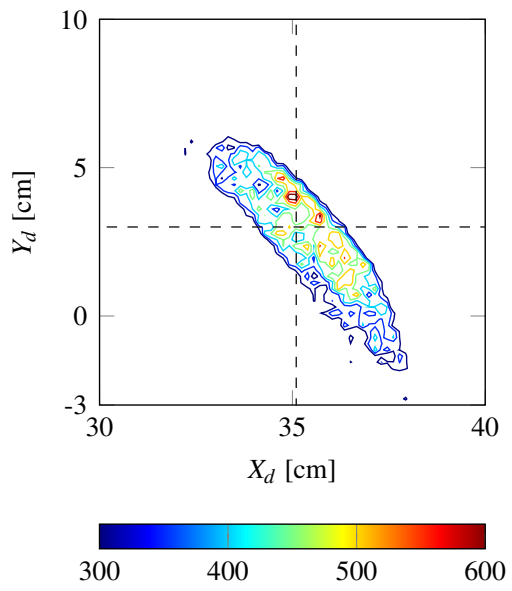

(d) One array

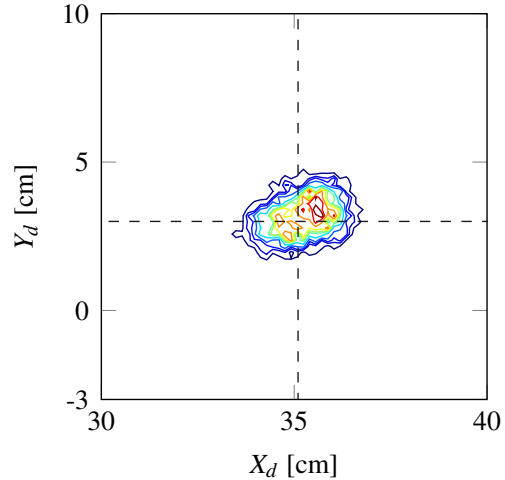

$1,000 \quad 2,000 \quad 3,000 \quad 4,000$

(e) Two arrays

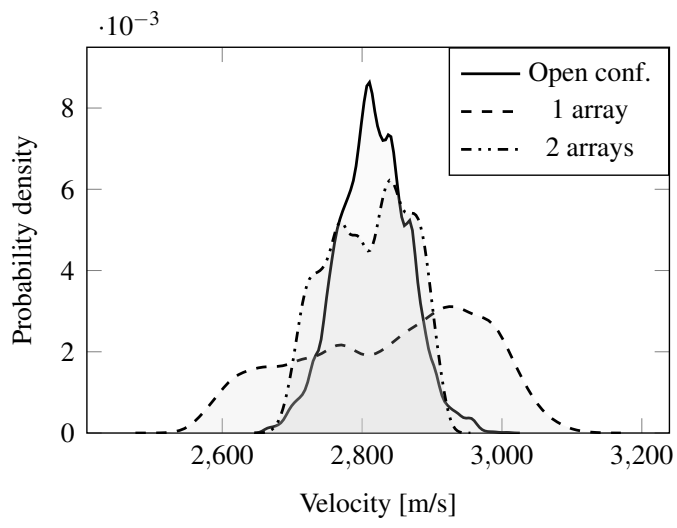

(f) Velocity reconstruction

Figure 7: Abaqus model in (a) and dimensions in (b) of the aerospace panel. Panels (c) to (e) show the resulting damage position reconstruction corresponding to the optimal configurations using the open, one-array and two-array configurations, respectively. The color bars indicate the probability density of the joint distribution. Panel (f) represents the wave propagation velocity reconstruction. The irregularities of contour lines in plots (c) to (e) could be improved using more AIMS samples at the cost of heavier computation. However they are shown to be enough for illustration and validation purposes. 
supported by the wave velocity reconstructions observed in Figure $7 \mathrm{f}$. where the posterior PDFs provided by the open and two-array configurations are more accurate (i.e. have less uncertainty) than the one with one-array configuration.

\section{Discussion}

\subsection{On the case studies results}

The proposed methodology for optimal sensor configuration in ultrasonic guided-wave based SHM has been illustrated in Section 3 using two case studies. In particular, the optimal number and locations of sensors have been shown to be sensitive to the choice of a particular inverse cost function. Thus, a proper inverse cost definition is key for an accurate and efficient SHM design. This was an expected result as a consequence of using the value instead of the amount of information as the optimality criterion. Note that the definition of the inverse cost function depends on the manufacturing and maintenance costs derived from the SHM system, and thus it is case specific. For instance, if the cost increases linearly with the number of sensors, a linearly decreasing inverse cost function could be assumed to be appropriate. To further explore the dependence of the optimization results on the inverse cost function, the implications of using different inverse cost functions in the optimal number of sensors are discussed here. In particular, Figure 8 shows two different but similar numerical inverse cost functions, namely $f_{1}(n)$ and $f_{2}(n)$, and their influence in the value of information curve with respect to the number of sensors for case study 1 . As is evident from the results in Figures $8 \mathrm{~b}$ and $8 \mathrm{~d}$, the optimal number of sensors varies from 5 to 9 sensors just by changing the inverse cost function from $f_{1}(n)$ to $f_{2}(n)$, respectively. This simple example reveals the significance of the inverse cost function in the optimal sensor configuration under the proposed methodology based on value of information.

The importance of the definition of the inverse cost function $f(n)$ is also revealed in case study 2. Figure 9 summarizes the EVI (recall Eq. (9) for each of the optimal configurations under the consideration of both $f_{0}(n)$ (recall Eq. (15)) and $f_{1}(n)$. As observed, the optimal solution under $f_{1}(n)$ provides higher EVI values, which is in agreement with the more accurate and efficient (i.e. using fewer sensors) damage reconstructions observed in Figures $7 \mathrm{c}$ to $7 \mathrm{e}$. Observe also that the EVI not only enables a rational criterion for optimal sensor configuration, but also establishes a measure to compare between several candidate inverse cost functions.

Nevertheless, this dependence upon the inverse cost function may arguably be seen as an additional complexity, which may lead to suboptimal results depending on priorities, e.g. whether or not the cost is more important than the amount of information gained. However, we show here that adopting the value instead of the amount of information provides a better identifiability of the optimal design point. This is manifested in Figure 10, where the KL divergence criterion is compared against the EVI criterion, taking the second case study using $f_{0}(n)$ and $\alpha=10$ as an example. As shown in the results, an optimal SHM design point would be barely identifiable using the amount of information criterion (Figure 10a) in view of the asymptotic behavior of the KL divergence. In contrast, the estimator of the EVI (i.e., $\left.J\left(\mathscr{C}_{o p t}^{n}\right)\right)$ shown in Figure 10 b reaches a globally identifiable optimum value, which greatly facilitates the optimization process. 


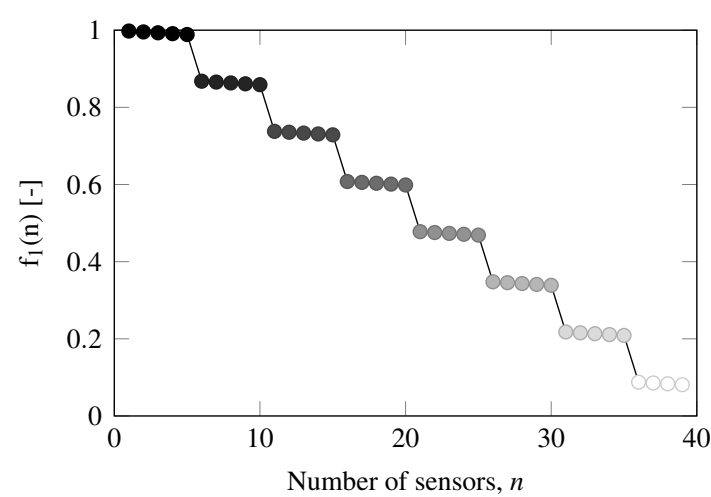

(a) Inverse cost function $f_{1}(n)$

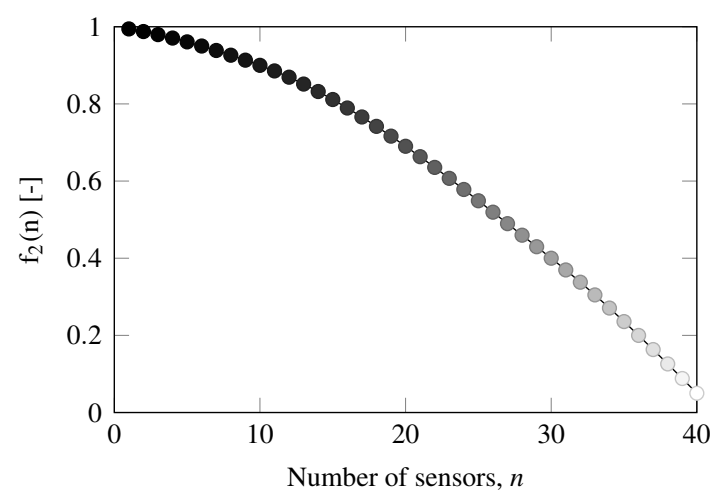

(c) Inverse cost function $f_{2}(n)$

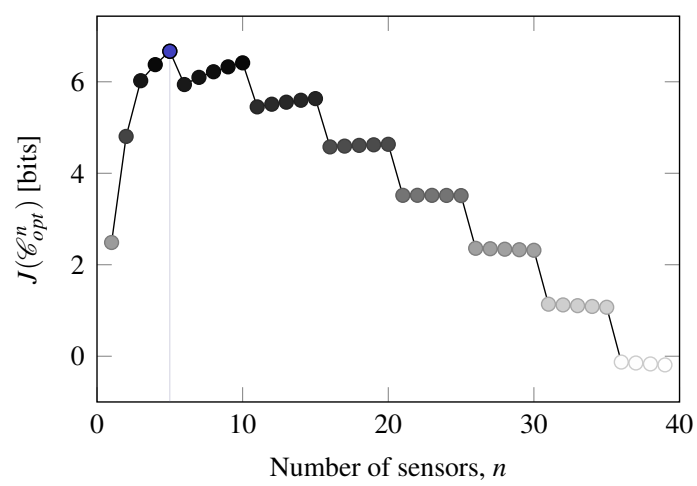

(b) Value of information for different $n$

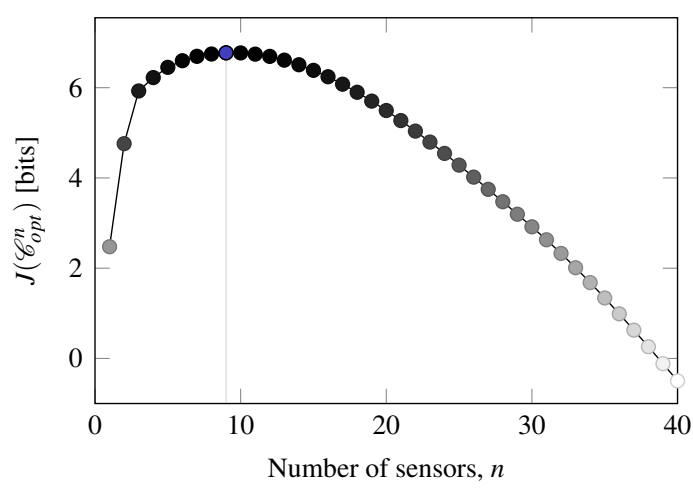

(d) Value of information for different $n$

Figure 8: Alternative inverse cost functions accounting for SHM systems using slots of 5 sensors (a) along with the resulting influence in the optimal sensor configuration in (b). In (c), a different function which is continuous rather than discrete with a similar trend to (a) leads to a different optimal sensor configuration in (d).

Note that the constant $\alpha$ included in Equation (1) plays an important role in modulating the EVI since it scales the relative cost component in Equation 97. However, in view of Figure 11a the rank between the candidate sensor positioning strategies results to be unaltered by this constant, where the EVI is calculated for $\alpha \in[0,1000]$ using $f_{1}(n)$ and the data obtained in case study 2 . Conversely, $\alpha$ is shown to influence the optimal number of sensors, as observed in Figure 11b In particular, $n_{\text {opt }}^{\prime \prime}$ tends to decrease as long as $\alpha$ increases as a consequence of the increasing cost of sensors in the context of the trade-off between information and cost given by Equation (9).

\subsection{On the extensibility of the method}

The proposed methodology provides the optimal sensor configuration for a specific area of possible damage occurrence based on a ToF model, which takes into account the position of sensors, actuators, damage, and the wave propagation velocity. Further, the resulting optimal configurations have proven efficiency in reconstructing the damage position. However, the ToF model lacks of advanced physics-related information such as the interaction of the ultrasonic guided-wave with the damage or the effect of attenuation in the localization in the damage. This prevents 


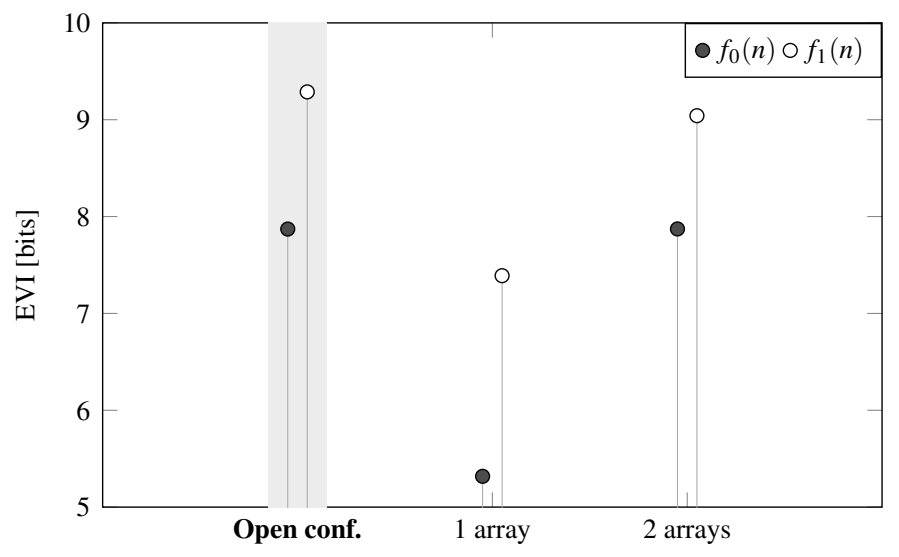

Figure 9: Results of the comparison in terms of EVI (circles) for the different sensor positioning strategies and inverse cost functions, namely $f_{0}(n)$ (gray symbols) and $f_{1}(n)$ (white symbols), for case study 2. Points on the upper part of the chart represent more informative alternatives.

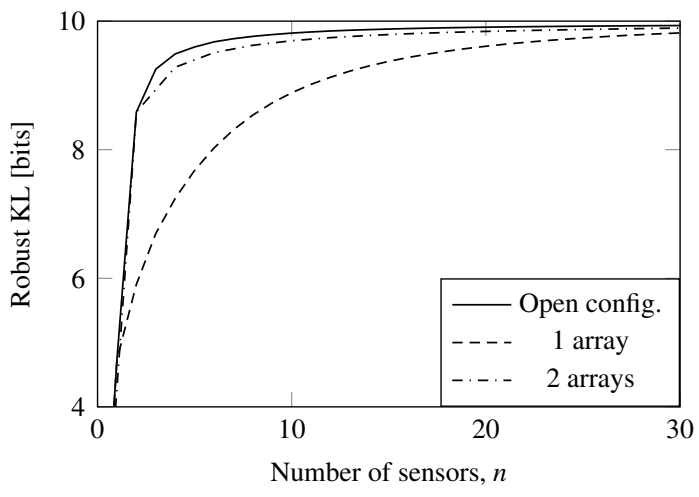

(a) Robust KL divergence criterion

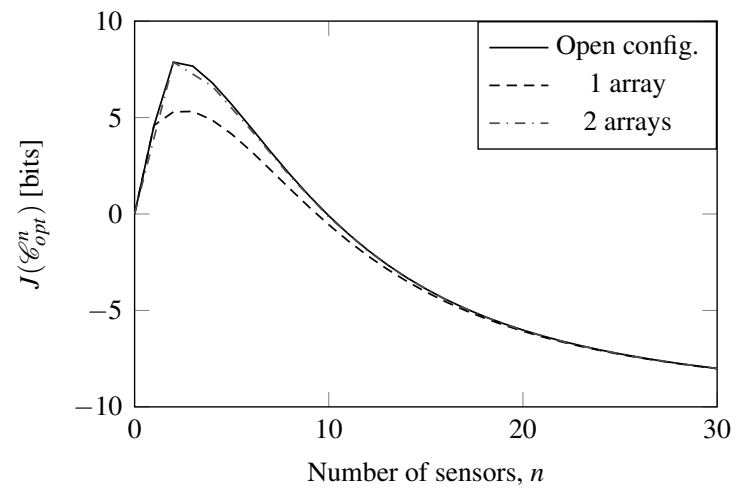

(b) EVI criterion

Figure 10: Comparison between the robust KL divergence over the area of possible damage occurrence and the EVI criterion using $f_{0}(n)$.

the proposed methodology from considering advanced damage features other than the position, such as damage mode and extent (e.g., delamination in composites), and from providing an optimal sensor configuration considering them. In order to consider these advanced damage features, a more complex and physics-based wave propagation model such as the hybrid wave and finite element methods [71] is required. The use of these models in the design of the optimal sensor configuration is still unfeasible due to their considerable computational cost. Therefore, the development of methods to alleviate such computational complexity, such as surrogate methods [72-76], constitutes a desirable extension of this work.

Furthermore, note that the proposed method seeks the optimal sensor configuration, assuming that the actuators are fixed in the structure. However, the position and number of actuators play an important role in the optimization problem. Besides, the sequential sensor placement algorithm using an exhaustive search may find a limitation in practice when considering very large areas of possible damage occurrence. In this context, an ongoing extension of the proposed methodology is the consideration of both sensors and actuators in the optimization problem considering 


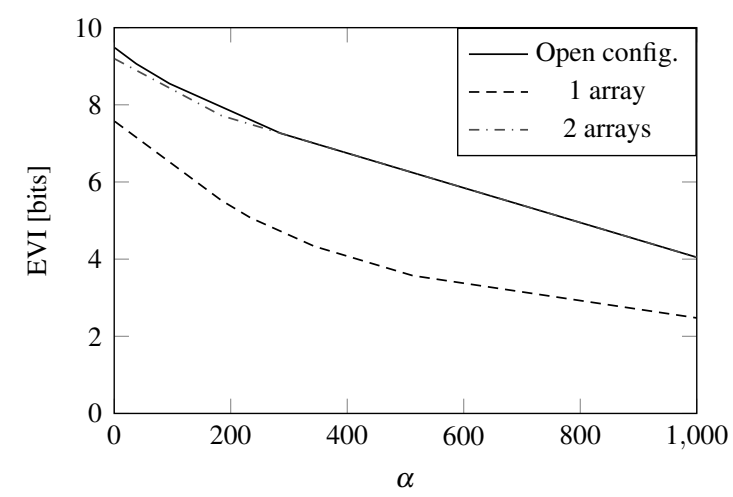

(a) EVI for each configuration

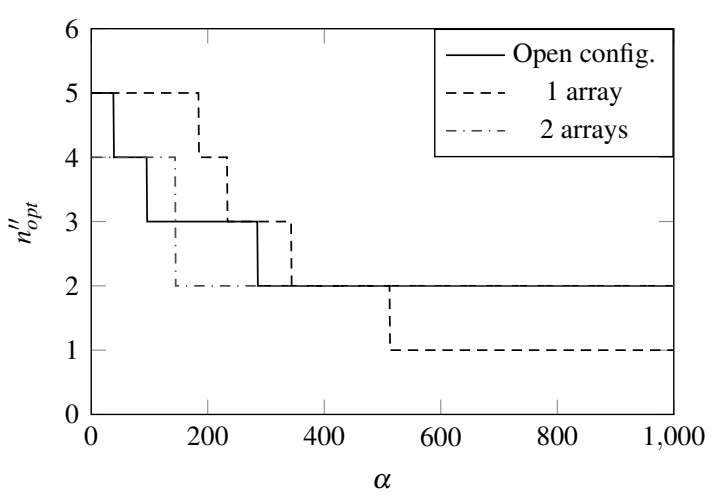

(b) Optimal number of sensors

Figure 11: Effect of $\alpha$ values in the EVI of each sensor configuration in (a) and its effect in the optimal number of sensors in (b).

large structures.

\section{Conclusions}

A novel optimal sensor configuration approach based on value of information is presented in this paper. The methodology is based on a Bayesian damage localization framework for optimal sensor placement, while the optimal number of sensors is obtained by assessing the expected value of information; thus, uncertainties coming from several sources are taken into consideration. The effectiveness in providing an optimal design point has been illustrated using two case studies considering both flat and stiffened plate structures, respectively. The following conclusions are drawn from this paper:

- The proposed approach provides a value of information-based framework that trades-off the amount of information and the cost of monitoring, giving a globally identifiable optimal design point.

- The open configuration provides the best damage reconstruction using the lower number of sensors due to its flexibility in positioning sensors freely over the structure;

- Based on the results, the two-array configuration provides more accurate damage reconstructions using less number of sensors than the one-array configuration.

- An accurate definition of the inverse cost function has been shown to be key for an unbiased optimal sensor configuration under the proposed methodology.

Further research work is needed to address (1) the extensibility of the proposed method using a physics-based model instead of the time of flight one used here, (2) the efficiency of the method when considering the optimization of both actuators and sensors in large plate-like structures, and (3) the optimality of the inverse cost function in different specific cases. 


\section{Acknowledgements}

This paper is part of the SAFE-FLY project that has received funding from the European Union's Horizon 2020 research and innovation programme under the Marie Skłodowska-Curie grant agreement No 721455. In addition, the authors are grateful for the access to the University of Nottingham High Performance Computing Facility and to the University of Granada for "ROBIN" grant [30.BF.66.11.01], which partially provides support to this work.

\section{References}

[1] M. Gerdes, D. Scholz, D. Galar, Effects of condition-based maintenance on costs caused by unscheduled maintenance of aircraft, Journal of Quality in Maintenance Engineering 22 (4) (2016) 394-417.

[2] J.-H. Shin, H.-B. Jun, On condition based maintenance policy, Journal of Computational Design and Engineering 2 (2) (2015) $119-127$.

[3] R. A. Howard, Information value theory, IEEE Transactions on Systems Science and Cybernetics 2 (1) (1966) $22-26$.

[4] R. Schlaifer, H. Raiffa, Applied Statistical Decision Theory, MIT Press, 1961.

[5] M. Pozzi, A. Der Kiureghian, Assessing the value of information for long-term structural health monitoring, in: Health monitoring of structural and biological systems 2011, Vol. 7984, International Society for Optics and Photonics, 2011, p. 79842W.

[6] A. Zitrou, T. Bedford, A. Daneshkhah, Robustness of maintenance decisions: Uncertainty modelling and value of information, Reliability Engineering \& System Safety 120 (2013) 60-71.

[7] D. Zonta, B. Glisic, S. Adriaenssens, Value of information: impact of monitoring on decision-making, Structural Control and Health Monitoring 21 (7) (2014) 1043-1056.

[8] K. Konakli, B. Sudret, M. H. Faber, Numerical investigations into the value of information in lifecycle analysis of structural systems, ASCEASME Journal of Risk and Uncertainty in Engineering Systems, Part A: Civil Engineering 2 (3) (2015) B4015007.

[9] D. Straub, Value of information analysis with structural reliability methods, Structural Safety 49 (2014) 75-85.

[10] D. Straub, E. Chatzi, E. Bismut, W. Courage, M. Döhler, M. H. Faber, J. Köhler, G. Lombaert, P. Omenzetter, M. Pozzi, et al., Value of information: A roadmap to quantifying the benefit of structural health monitoring, in: ICOSSAR-12th International Conference on Structural Safety \& Reliability, 2017.

[11] S. Thöns, M. H. Faber, Assessing the value of structural health monitoring, in: 11th International Conference on Structural Safety and Reliability, Balkema Publishers, AA/Taylor \& Francis The Netherlands, 2013, pp. 2543-2550.

[12] S. Thöns, R. Schneider, M. H. Faber, Quantification of the value of structural health monitoring information for fatigue deteriorating structural systems, in: 12th International Conference on Applications of Statistics and Probability in Civil Engineering (ICASP12), 2015, pp. 1-8.

[13] J. Qin, S. Thöns, M. H. Faber, On the value of SHM in the context of service life integrity management, in: 12th International Conference on Applications of Statistics and Probability in Civil Engineering (ICASP12), 2015, pp. 1-8.

[14] S. Thöns, On the Value of Monitoring Information for the Structural Integrity and Risk Management, Computer-Aided Civil and Infrastructure Engineering 33 (1) (2018) 79-94.

[15] C. Malings, M. Pozzi, Value of information for spatially distributed systems: Application to sensor placement, Reliability Engineering \& System Safety 154 (2016) 219-233.

[16] C. Malings, M. Pozzi, Submodularity issues in value-of-information-based sensor placement, Reliability Engineering \& System Safety 183 (2019) 93-103.

[17] W. J. Staszewski, K. Worden, R. Wardle, G. R. Tomlinson, Fail-safe sensor distributions for impact detection in composite materials, Smart Materials and Structures 9 (3) (2000) 298.

[18] K. Worden, W. Staszewski, Impact location and quantification on a composite panel using neural networks and a genetic algorithm, Strain 36 (2) (2000) 61-68.

[19] K. Worden, A. Burrows, Optimal sensor placement for fault detection, Engineering structures 23 (8) (2001) 885-901. 
[20] V. Mallardo, M. Aliabadi, Z. S. Khodaei, Optimal sensor positioning for impact localization in smart composite panels, Journal of Intelligent Material Systems and Structures 24 (5) (2013) 559-573.

[21] M. De Stefano, M. Gherlone, M. Mattone, M. Di Sciuva, K. Worden, Optimum sensor placement for impact location using trilateration, Strain 51 (2) (2015) 89-100.

[22] F. Y. Lin, P.-L. Chiu, A near-optimal sensor placement algorithm to achieve complete coverage-discrimination in sensor networks, IEEE Communications Letters 9 (1) (2005) 43-45.

[23] P. Blanloeuil, N. A. Nurhazli, M. Veidt, Particle swarm optimization for optimal sensor placement in ultrasonic SHM systems, in: Nondestructive Characterization and Monitoring of Advanced Materials, Aerospace, and Civil Infrastructure 2016, Vol. 9804, International Society for Optics and Photonics, 2016, pp. $9804-9804-11$.

[24] H. Gao, J. Rose, Ultrasonic sensor placement optimization in structural health monitoring using evolutionary strategy, in: AIP Conference Proceedings, Vol. 820, AIP, 2006, pp. 1687-1693.

[25] J. F. Markmiller, F.-K. Chang, Sensor network optimization for a passive sensing impact detection technique, Structural Health Monitoring 9 (1) (2010) 25-39.

[26] H. Guo, L. Zhang, L. Zhang, J. Zhou, Optimal placement of sensors for structural health monitoring using improved genetic algorithms, Smart Materials and Structures 13 (3) (2004) 528.

[27] M. Azarbayejani, A. El-Osery, K. Choi, M. R. Taha, A probabilistic approach for optimal sensor allocation in structural health monitoring, Smart Materials and Structures 17 (5) (2008) 055019.

[28] M. Thiene, Z. Sharif-Khodaei, M. Aliabadi, Optimal sensor placement for damage detection based on ultrasonic guided wave, in: Key Engineering Materials, Vol. 665, Trans Tech Publ, 2016, pp. 269-272.

[29] M. Thiene, Z. S. Khodaei, M. Aliabadi, Optimal sensor placement for maximum area coverage (MAC) for damage localization in composite structures, Smart Materials and Structures 25 (9) (2016) 095037.

[30] Z. S. Khodaei, M. Aliabadi, An optimization strategy for best sensor placement for damage detection and localization in complex composite structures, in: 8th European Workshop On Structural Health Monitoring (EWSHM 2016), 2016, pp. 5-8.

[31] H. Tarhini, R. Itani, M. A. Fakih, S. Mustapha, Optimization of piezoelectric wafer placement for structural health-monitoring applications, Journal of Intelligent Material Systems and Structures (2018) 1045389X18799204.

[32] M. Salmanpour, Z. Sharif Khodaei, M. Aliabadi, Transducer placement optimisation scheme for a delay and sum damage detection algorithm, Structural Control and Health Monitoring 24 (4)

[33] J. L. Beck, C. Papadimitriou, S.-K. Au, M. W. Vanik, Entropy-based optimal sensor location for structural damage detection, in: Smart Structures and Materials 1998: Smart Systems for Bridges, Structures, and Highways, Vol. 3325, International Society for Optics and Photonics, 1998, pp. 161-173.

[34] C. Papadimitriou, J. L. Beck, S.-K. Au, Entropy-based optimal sensor location for structural model updating, Journal of Vibration and Control 6 (5) (2000) 781-800.

[35] C. Papadimitriou, Optimal sensor placement methodology for parametric identification of structural systems, Journal of Sound and Vibration 278 (4-5) (2004) 923-947.

[36] C. Papadimitriou, G. Lombaert, The effect of prediction error correlation on optimal sensor placement in structural dynamics, Mechanical Systems and Signal Processing 28 (2012) 105-127.

[37] J. Zhang, K. Maes, G. De Roeck, E. Reynders, C. Papadimitriou, G. Lombaert, Optimal sensor placement for multi-setup modal analysis of structures, Journal of Sound and Vibration 401 (2017) 214-232.

[38] X. Huan, Y. M. Marzouk, Simulation-based optimal Bayesian experimental design for nonlinear systems, Journal of Computational Physics 232 (1) (2013) 288-317.

[39] C. Argyris, C. Papadimitriou, P. Panetsos, Bayesian optimal sensor placement for modal identification of civil infrastructures, Journal of Smart Cities 2 (2) (2017) 69-86.

[40] G. Capellari, E. Chatzi, S. Mariani, Optimal sensor placement through Bayesian experimental design: effect of measurement noise and 
number of sensors, in: Multidisciplinary Digital Publishing Institute Proceedings, Vol. 1, 2017, p. 41.

[41] C. Argyris, S. Chowdhury, V. Zabel, C. Papadimitriou, Bayesian optimal sensor placement for crack identification in structures using strain measurements, Structural Control and Health Monitoring 25 (5) (2018) e2137.

[42] G. Capellari, E. Chatzi, S. Mariani, Cost-benefit optimization of structural health monitoring sensor networks, Sensors 18 (7) (2018) 2174.

[43] W. M. Said, W. J. Staszewski, Optimal sensor location for damage detection using mutual information, Signal Processing and Composites (2001) 428-435.

[44] G. Capellari, E. Chatzi, S. Mariani, Structural health monitoring sensor network optimization through Bayesian experimental design, ASCEASME Journal of Risk and Uncertainty in Engineering Systems, Part A: Civil Engineering 4 (2) (2018) 04018016.

[45] K. Chaloner, I. Verdinelli, Bayesian experimental design: A review, Statistical Science (1995) 273-304.

[46] S. Cantero-Chinchilla, J. Chiachío, M. Chiachío, D. Chronopoulos, A. Jones, A robust Bayesian methodology for damage localization in plate-like structures using ultrasonic guided-waves, Mechanical Systems and Signal Processing 122 (2019) 192-205.

[47] E. B. Flynn, M. D. Todd, P. D. Wilcox, B. W. Drinkwater, A. J. Croxford, Maximum-likelihood estimation of damage location in guided-wave structural health monitoring, in: Proceedings of the Royal Society of London A: Mathematical, Physical and Engineering Sciences, Vol. 467, The Royal Society, 2011, pp. 2575-2596.

[48] K. J. Ryan, Estimating expected information gains for experimental designs with application to the random fatigue-limit model, Journal of Computational and Graphical Statistics 12 (3) (2003) 585-603.

[49] L. Cohen, Time-frequency Analysis: Theory and Applications, Prentice-Hall, Inc., Upper Saddle River, NJ, USA, 1995.

[50] N. E. Huang, Z. Shen, S. R. Long, M. C. Wu, H. H. Shih, Q. Zheng, N.-C. Yen, C. C. Tung, H. H. Liu, The empirical mode decomposition and the Hilbert spectrum for nonlinear and non-stationary time series analysis, in: Proceedings of the Royal Society of London A: mathematical, physical and engineering sciences, Vol. 454, The Royal Society, 1998, pp. 903-995.

[51] C. K. Chui, An introduction to wavelets, Academic Press Professional, Inc., San Diego, CA, USA, 1992.

[52] C. Bao, H. Hao, Z.-X. Li, X. Zhu, Time-varying system identification using a newly improved HHT algorithm, Computers \& Structures 87 (23-24) (2009) 1611-1623.

[53] J.-B. Ihn, F.-K. Chang, Pitch-catch active sensing methods in structural health monitoring for aircraft structures, Structural Health Monitoring 7 (1) (2008) 5-19.

[54] J. L. Beck, Bayesian system identification based on probability logic, Structural Control and Health Monitoring 17 (7) (2010) $825-847$.

[55] E. T. Jaynes, Information theory and statistical mechanics, Physical review 106 (4) (1957) 620.

[56] A. Tarantola, Inverse problem theory and methods for model parameter estimation, Vol. 89, SIAM, 2005.

[57] G. Rus, J. Chiachío, M. Chiachío, Logical inference for inverse problems, Inverse Problems in Science and Engineering 24 (3) (2016) $448-464$.

[58] F. Liang, C. Liu, J. Chuanhai, Advanced Markov Chain Monte Carlo Methods, Wiley Online Library, 2010.

[59] A. Krause, A. Singh, C. Guestrin, Near-optimal sensor placements in gaussian processes: Theory, efficient algorithms and empirical studies, Journal of Machine Learning Research 9 (Feb) (2008) 235-284.

[60] T.-H. Yi, H.-N. Li, Methodology developments in sensor placement for health monitoring of civil infrastructures, International Journal of Distributed Sensor Networks 8 (8) (2012) 612726.

[61] G. Aranguren, P. Monje, V. Cokonaj, E. Barrera, M. Ruiz, Ultrasonic wave-based structural health monitoring embedded instrument, Review of Scientific Instruments 84 (12) (2013) 125106

[62] A. Alcaide, E. Barrera, M. Ruiz, G. Aranguren, Damage detection on Aerospace structures using PAMELA SHM System, in: 6th International Symposium on NDT in Aerospace, Madrid, 2014.

[63] ABAQUS, Abaqus Documentation, Dassault Systèmes, Providence, RI, USA (2016).

[64] D. Alleyne, P. Cawley, A two-dimensional Fourier transform method for the measurement of propagating multimode signals, The Journal of the Acoustical Society of America 89 (3) (1991) 1159-1168.

[65] Z. Su, L. Ye, Y. Lu, Guided Lamb waves for identification of damage in composite structures: A review, Journal of Sound and Vibration 
[66] J. L. Beck, K. M. Zuev, Asymptotically independent Markov sampling: a new Markov chain Monte Carlo scheme for Bayesian inference, International Journal for Uncertainty Quantification 3 (5).

[67] K. M. Zuev, J. L. Beck, Global optimization using the asymptotically independent Markov sampling method, Computers \& Structures 126 (2013) 107-119.

[68] M. Chiachío, J. Chiachío, G. Rus, J. L. Beck, Predicting fatigue damage in composites: A Bayesian framework, Structural Safety 51 (2014) $57-68$.

[69] A. Gelman, G. Roberts, W. Gilks, Efficient Metropolis jumping rules, Bayesian Statistics 5 (1996) 599-608

[70] G. Roberts, J. Rosenthal, Optimal scaling for various Metropolis-Hastings algorithms, Statistical Science 16 (4) (2001) $351-367$.

[71] B. R. Mace, E. Manconi, Modelling wave propagation in two-dimensional structures using finite element analysis, Journal of Sound and Vibration 318 (4-5) (2008) 884-902.

[72] R. Yondo, E. Andrés, E. Valero, A review on design of experiments and surrogate models in aircraft real-time and many-query aerodynamic analyses, Progress in Aerospace Sciences.

[73] A. Giunta, L. Watson, A comparison of approximation modeling techniques-Polynomial versus interpolating models, in: 7th AIAA/USAF/NASA/ISSMO Symposium on Multidisciplinary Analysis and Optimization, 1998, p. 4758.

547 [74] J. Sacks, W. J. Welch, T. J. Mitchell, H. P. Wynn, Design and analysis of computer experiments, Statistical Science (1989) $409-423$.

548 [75] R. G. Ghanem, P. D. Spanos, Stochastic finite elements: a spectral approach, Springer-Verlag, New York, 1991.

549 [76] N. Wiener, The homogeneous chaos, American Journal of Mathematics 60 (4) (1938) 897-936. 